Supporting Information for:

\title{
Modular Attachment of Appended Boron Lewis Acids to a Ruthenium Pincer Catalyst: Metal-Ligand Cooperativity Enables Selective Alkyne Hydrogenation
}

\author{
Kuei-Nin T. Tseng, Jeff W. Kampf, and Nathaniel K. Szymczak* \\ Department of Chemistry, University of Michigan, 930 N. University Ave., Ann Arbor, MI \\ 48109, United States \\ Corresponding Author: nszym@umich.edu
}

\section{Table of Contents}

General Considerations

Alkene Hydrogenation Reactions Catalyzed by $\mathrm{HRu}(\mathrm{bMepi})\left(\mathrm{PPh}_{3}\right)_{2}$

General Procedure for Catalytic Hydrogenation Reactions

Table S1. Hydrogenation of Alkenes Catalyzed by Ru(CH9BBNMepi) $\mathrm{PPh}_{3}$

Preparation of Bifunctional Ruthenium Complexes with Appended 9-BBN, BCat, and BPin

Solution Characterization of $\mathrm{Ru}(\mathrm{CH} 9 \mathrm{BBNMepi}) \mathrm{PPh}_{3}$

NMR spectra of $\mathrm{Ru}(\mathrm{CH} 9 \mathrm{BBNMepi}) \mathrm{PPh}_{3}$

NMR spectra of $\mathrm{HRu}\left(\mathrm{CH}_{2} \mathrm{BCatMepi}\right) \mathrm{PPh}_{3}$

NMR spectra of $\mathrm{Ru}\left(\mathrm{CBPin}_{2} \mathrm{Mepi}\right) \mathrm{PPh}_{3}$

NMR spectra of $\mathrm{HRu}\left(\mathrm{CH}_{2} 9 \mathrm{BBNMepi}\right)\left(\mathrm{PPh}_{3}\right) \mathrm{CO}$

Competition Experiments between 1-Octyne and 1-Octene

NMR spectra of competition experiments using $\mathrm{HRu}\left(\mathrm{CH}_{2} \mathrm{BCatMepi}\right) \mathrm{PPh}_{3}$

NMR spectra of competition experiments using $\mathrm{HRu}(\mathrm{bMepi})\left(\mathrm{PPh}_{3}\right)_{2}$

NMR spectra of catalytic hydrogenation reactions of alkynes

NMR spectra of $\mathrm{Ru}\left(\mathrm{CH} 9 \mathrm{BBNMepi)} \mathrm{PPh}_{3}\right.$ under $\mathrm{H}_{2}$

2D NMR Experiments with Ru(CH9BBNMepi) $\mathrm{PPh}_{3}$ 


\section{General Considerations}

All manipulations were conducted under a nitrogen atmosphere on a Schlenk manifold or in a glovebox using standard Schlenk techniques, unless otherwise stated. All reagents were purchased from commercial vendors. Pinacolborane (HBPin; Aldrich), borabicyclo[3.3.1]nonane (9-BBN; Aldrich), and catecholborane (HBCat; Aldrich) were used without further purification. Substrates (1-octene, Aldrich; cyclooctene, Aldrich; styrene, Aldrich; 4-octyne, Aldrich; 1octyne, Aldrich; cis-4-octene, Alfa Aesar) were distilled from $\mathrm{CaH}_{2}$ under a nitrogen atmosphere, and then stored over $3 \AA$ molecular sieves for $24 \mathrm{~h}$. The following compounds were synthesized according to literature methods: $\mathrm{HRu}(\mathrm{bMepi})\left(\mathrm{PPh}_{3}\right)_{2}(\mathbf{1}),{ }^{1}\left[\mathrm{Ru}\left(\mathrm{CH}_{2} \mathrm{Mepi}\right) \mathrm{PPh}_{3}\right]_{2}(\mathbf{2}),{ }^{2}$ $(9-\mathrm{BBN}) \mathrm{CH}_{2} \mathrm{CH}_{2} \mathrm{Ph},{ }^{3}$ and $\mathrm{HRu}\left(\mathrm{b}^{i} \mathrm{Prpi}\right)\left(\mathrm{PPh}_{3}\right)_{2}(\mathbf{1 0}){ }^{5}$ The $3 \AA$ Molecular sieves were dried at $250{ }^{\circ} \mathrm{C}$ under dynamic vacuum for $24 \mathrm{~h}$. Dichloromethane (DCM), diethyl ether $\left(\mathrm{Et}_{2} \mathrm{O}\right)$, pentane, and benzene $\left(\mathrm{C}_{6} \mathrm{H}_{6}\right)$ were purified using a Glass Contour solvent purification system consisting of a copper catalyst, neutral alumina, and activated molecular sieves, then passed through an in-line, $2 \mu \mathrm{m}$ filter immediately before being dispensed. Toluene (PhMe) was sparged using nitrogen and then stored over $3 \AA$ molecular sieves for at least $24 \mathrm{~h}$.

NMR spectra were recorded on Varian Inova 500, Varian MR400, Varian vnmrs 500, and Varian vnmrs 700 spectrometers at room temperature. ${ }^{1} \mathrm{H}$ and ${ }^{13} \mathrm{C}$ shifts are reported in parts per million (ppm) relative to TMS, with the residual solvent peak used as an internal reference. ${ }^{31} \mathrm{P}$ NMR spectra were referenced on a unified scale to their respective ${ }^{1} \mathrm{H}$ NMR spectra and collected using 64-128 transients. The following abbreviations are reported as follows: broad (br), singlet (s), doublet (d), doublet of doublets (dd), triplet (t), quartet (q), multiplet (m), methyl (Me), and triphenylphosphine $\left(\mathrm{PPh}_{3}\right) .{ }^{13} \mathrm{C} \mathrm{NMR}$ resonances were observed as singlets unless otherwise stated. Solid-state IR spectra were collected using a Nicolet iS10 spectrometer equipped with a diamond attenuated total reflectance (ATR) accessory. Elemental analyses were performed by Midwest Microlab, LLC. 


\section{Alkene Hydrogenation Reactions Catalyzed by $\mathrm{HRu}(\mathrm{bMepi})\left(\mathrm{PPh}_{3}\right)_{2}$}

Alkenes $(0.1 \mathrm{mmol})$ were added to a J. Young NMR tube containing a $\mathrm{C}_{6} \mathrm{D}_{6}$ solution of 1 (1 mol\%, $0.001 \mathrm{mmol}, 0.9 \mathrm{mg}$ ). The J. Young NMR tube was capped with a Teflon key and charged with 30 psig of $\mathrm{H}_{2}$. After $6 \mathrm{~h}$ at $80{ }^{\circ} \mathrm{C}$, the conversions were determined by ${ }^{1} \mathrm{H} \mathrm{NMR}$ spectroscopy against phenyltrimethylsilane as an internal standard.

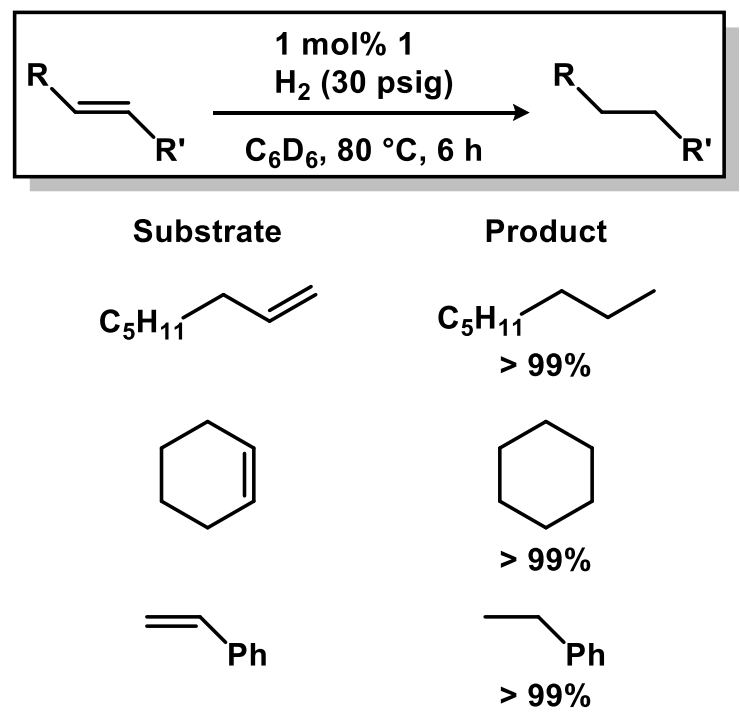




\section{General Procedure for Catalytic Hydrogenation Reactions}

Alkynes or alkenes $(0.1 \mathrm{mmol})$ were added to a J. Young NMR tube containing a $\mathrm{C}_{6} \mathrm{D}_{6}$ solution of $1 \mathrm{~mol} \%$ of Ru complexes $(0.001 \mathrm{mmol} ; \mathbf{1}, 0.9 \mathrm{mg} ; \mathbf{3}, 0.8 \mathrm{mg} ; \mathbf{5}, 0.8 \mathrm{mg})$. The J. Young NMR tube was capped with a Teflon key and charged with 30 psig of $\mathrm{H}_{2}$. After $2-8 \mathrm{~h}$ at $80{ }^{\circ} \mathrm{C}$, the products and yields were determined by ${ }^{1} \mathrm{H}$ NMR spectroscopy against phenyltrimethylsilane as an internal standard. The identity of the product was confirmed by comparison to the measured NMR spectra of authentic compounds.

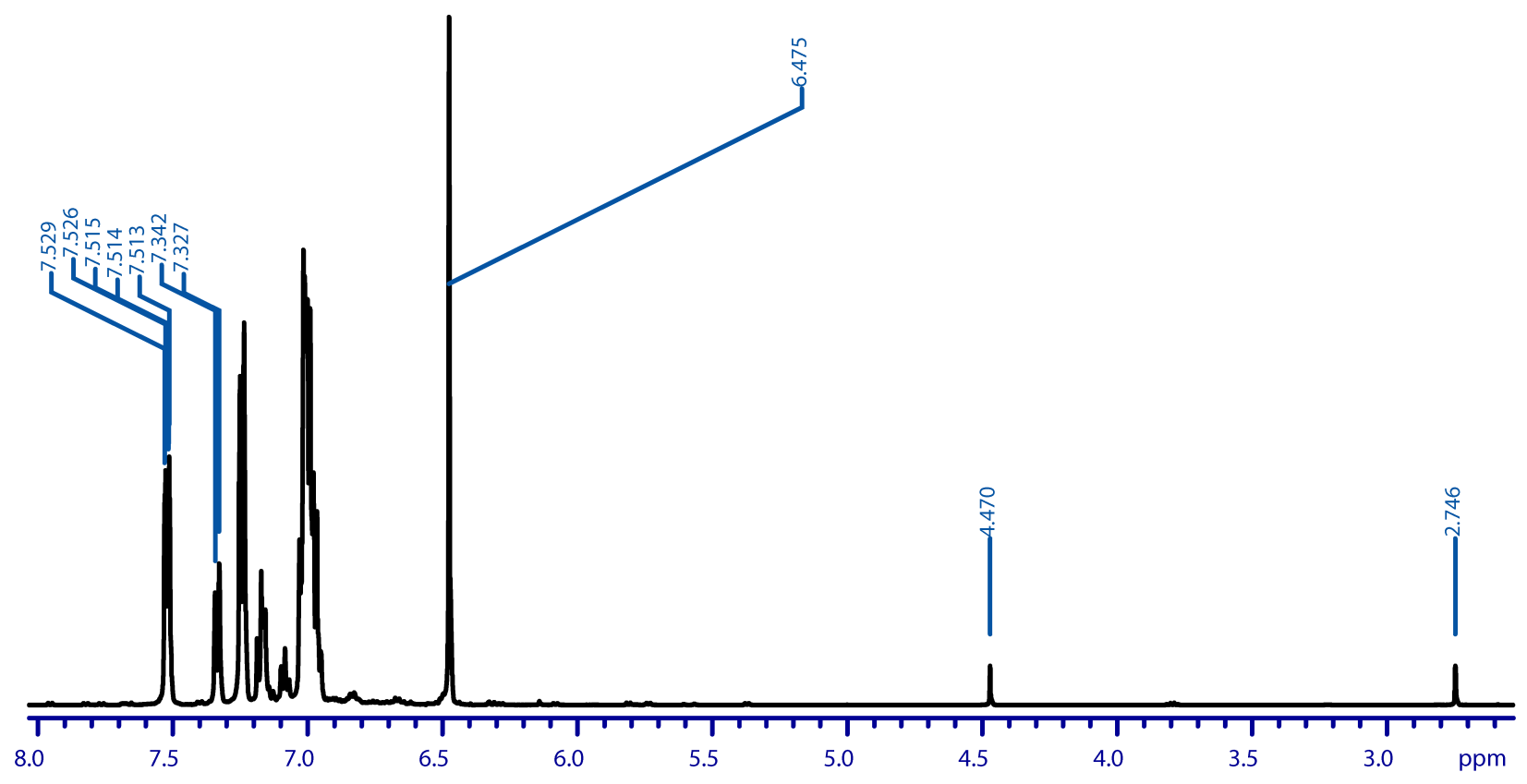

Figure S1. Representative ${ }^{1} \mathrm{H}$ NMR spectrum $\left(500 \mathrm{MHz}, \mathrm{C}_{6} \mathrm{D}_{6}\right)$ of catalytic hydrogenation of diphenylacetylene. The spectrum was referenced to $\mathrm{H}_{2}$ at $4.47 \mathrm{ppm}$. For clarity, only the peak values used to identify the products have been marked: $\delta 7.52$ (d, 4H, diphenylacetylene), 7.33 (d, 4H, E-stilbene), 6.48 (s, 2H, Z-stilbene), 2.75 (s, 4H, diphenylethane). 
Table S1. Hydrogenation of Alkenes Catalyzed by Ru(CH9BBNMepi)PPh

\begin{tabular}{|c|c|c|c|}
\hline \multirow[b]{2}{*}{ entry } & \multicolumn{2}{|c|}{$\begin{array}{c}1 \mathrm{~mol} \% 5 \\
\mathrm{H}_{2} \text { (30 psig) }\end{array}$} & \\
\hline & substrate & product & yield $(\%)$ \\
\hline 1 & cis-4-octene & octane & 100 \\
\hline 2 & 1-octene & octane & 100 \\
\hline 3 & cyclooctene & cyclooctane & 100 \\
\hline 4 & styrene & ethylbenzene & 100 \\
\hline
\end{tabular}




\section{General Procedure for Kinetic Experiments}

The observed reaction rates were obtained using the method of initial rates. Following the general procedure for catalytic hydrogenation reactions, the conversion of diphenylacetylene to $Z$-stilbene at $80{ }^{\circ} \mathrm{C}$ for $10 \mathrm{~min}$ was monitored by ${ }^{1} \mathrm{H}$ NMR spectroscopy. The J. Young NMR tube was placed in an oil bath preheated to $80^{\circ} \mathrm{C}$. After $2 \mathrm{~min}$, the NMR tube was removed from the oil bath and the ${ }^{1} \mathrm{H}$ NMR spectrum was acquired. This was repeated four more times to plot the kinetic trace in order to determine the initial rate. To confirm reproducibility and determine the initial rate, all kinetic experiments were performed in triplicate.

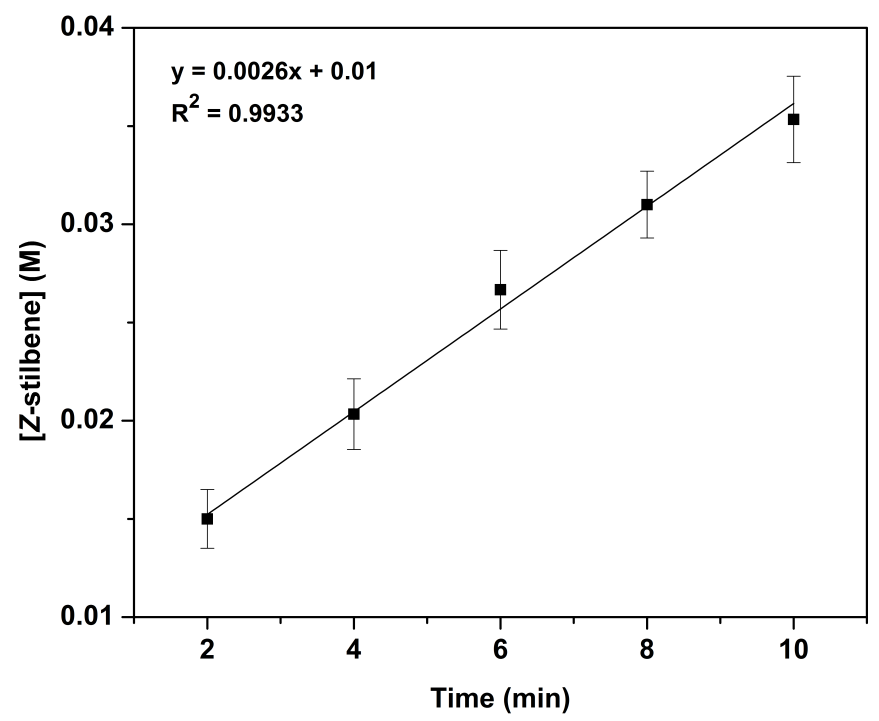

Figure S2. Rate of diphenylacetylene hydrogenation catalyzed by $\mathrm{Ru}(\mathrm{CH} 9 \mathrm{BBNMepi}) \mathrm{PPh}_{3}$ (5). 


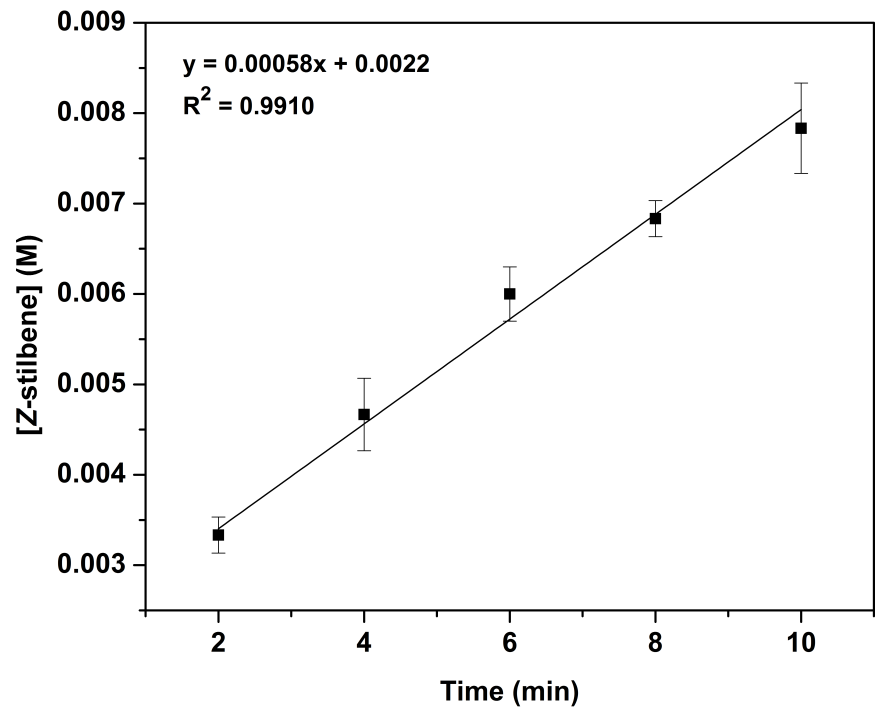

Figure S3. Rate of diphenylacetylene hydrogenation catalyzed by $\mathrm{HRu}\left(\mathrm{CH}_{2} \mathrm{BCatMepi}\right) \mathrm{PPh}_{3}(\mathbf{3})$.

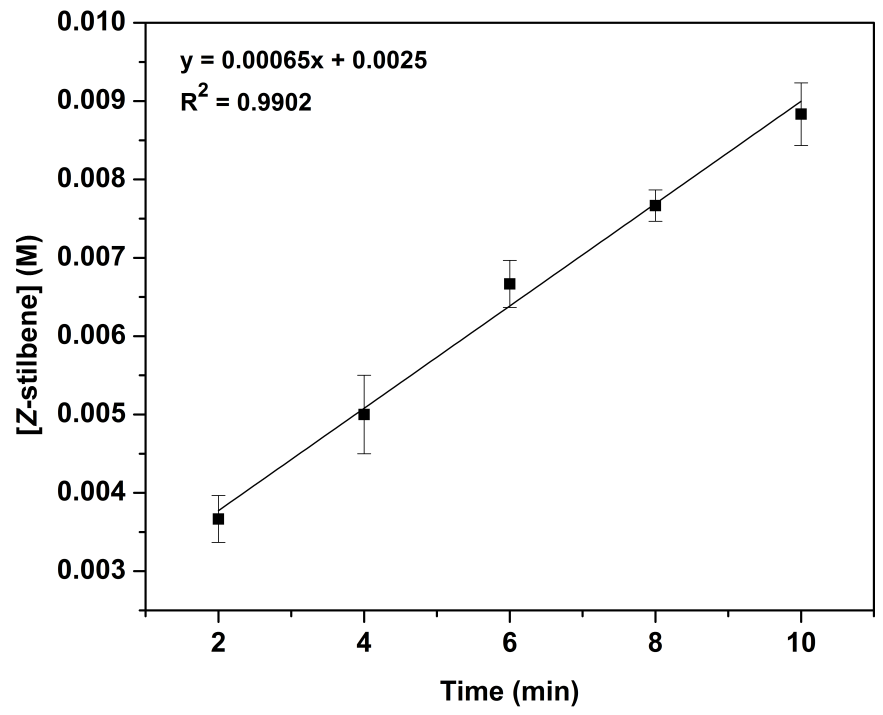

Figure S4. Rate of diphenylacetylene hydrogenation catalyzed by $\mathrm{HRu}(\mathrm{bMepi})\left(\mathrm{PPh}_{3}\right)_{2}(\mathbf{1})$. 


\section{Preparation of Bifunctional Ruthenium Complexes with Appended 9-BBN, BCat, and BPin}

$\mathbf{R u}\left(\mathbf{C H 9 B B N M e p i )} \mathbf{P P h}_{3}\right.$ (5). $\mathrm{C}_{6} \mathrm{H}_{6}(10 \mathrm{~mL})$ was added to a $20 \mathrm{~mL}$ vial containing $\left[\mathrm{Ru}\left(\mathrm{CH}_{2} \mathrm{Mepi}\right) \mathrm{PPh}_{3}\right]_{2}(98 \mathrm{mg}, 0.0571 \mathrm{mmol}), 9-\mathrm{BBN}$ (34.7 mg, $\left.0.142 \mathrm{mmol}\right)$, and a stir bar. The reaction solution was allowed to stir at $80{ }^{\circ} \mathrm{C}$ for $20 \mathrm{~h}$. After the solution cooled to room temperature, the $\mathrm{C}_{6} \mathrm{H}_{6}$ solvent was removed under vacuum and the crude product was washed with pentane $(4 \times 20 \mathrm{~mL})$. Evaporation of the volatiles under vacuum afforded the product as a dark green powder. Crystals were obtained from vapor diffusion of pentane into a $\mathrm{C}_{6} \mathrm{H}_{6}$ solution at room temperature. Yield: $90 \mathrm{mg}(78 \%) .{ }^{1} \mathrm{H} \mathrm{NMR}\left(500 \mathrm{MHz}, \mathrm{CD}_{2} \mathrm{Cl}_{2}\right): \delta 7.95\left(\mathrm{~d}, J_{\mathrm{HH}}=7.5 \mathrm{~Hz}\right.$, $1 \mathrm{H}), 7.60-7.52(\mathrm{~m}, 4 \mathrm{H}), 7.46\left(\mathrm{t}, J_{\mathrm{HH}}=7.5 \mathrm{~Hz}, 1 \mathrm{H}\right), 7.37\left(\mathrm{t}, J_{\mathrm{HH}}=7.5 \mathrm{~Hz}, 1 \mathrm{H}\right), 7.17\left(\mathrm{t}, J_{\mathrm{HH}}=7.5\right.$ $\left.\mathrm{Hz}, 3 \mathrm{H}, \mathrm{PPh}_{3}\right), 7.02-6.96(\mathrm{~m}, 7 \mathrm{H}), 6.85\left(\mathrm{t}, J_{\mathrm{HH}}=8.5 \mathrm{~Hz}, 6 \mathrm{H}, \mathrm{PPh}_{3}\right), 6.69\left(\mathrm{~d}, J_{\mathrm{HH}}=7.5 \mathrm{~Hz}, 1 \mathrm{H}\right)$, $6.60\left(\mathrm{~d}, J_{\mathrm{HH}}=5.5 \mathrm{~Hz}, 1 \mathrm{H}\right), 2.14\left(\mathrm{br} \mathrm{d}, J_{\mathrm{HH}}=15 \mathrm{~Hz}, 1 \mathrm{H}\right), 2.00(\mathrm{~s}, 3 \mathrm{H}, \mathrm{Me}), 1.80-1.67(\mathrm{~m}, 2 \mathrm{H})$, 1.59-1.52 (m, 2H), 1.42-1.17 (m, 5H), 0.99 (br t, $\left.J_{\mathrm{HH}}=6.8 \mathrm{~Hz}, 1 \mathrm{H}\right)$ 0.52-0.41 (br m, $\left.2 \mathrm{H}\right)$, $-1.16(\mathrm{~s}, 1 \mathrm{H}),-3.5$ (br s, $1 \mathrm{H}) .{ }^{13} \mathrm{C}\left\{{ }^{1} \mathrm{H}\right\}\left(176 \mathrm{MHz}, \mathrm{CD}_{2} \mathrm{Cl}_{2}\right): \delta 172.37,153.03,141.63,140.45$, $134.94,134.59,133.64\left(\mathrm{~d}, J_{\mathrm{CP}}=9.5 \mathrm{~Hz}\right), 133.44,129.69,128.97,128.70,128.33\left(\mathrm{~d}, J_{\mathrm{CP}}=8.4\right.$ $\mathrm{Hz}), 125.86,120.35,119.05,35.67$ (br), 34.29, 34.07, 33.83, 33.64, 27.17 (br), 25.55. ${ }^{31} \mathrm{P}\left\{{ }^{1} \mathrm{H}\right\}$ NMR (202 MHz, $\left.\mathrm{CD}_{2} \mathrm{Cl}_{2}\right): \delta 44.6$ (br s, $\left.\mathrm{PPh}_{3}\right) .{ }^{31} \mathrm{P}\left\{{ }^{1} \mathrm{H}\right\} \mathrm{NMR}\left(202 \mathrm{MHz}, \mathrm{CD}_{2} \mathrm{Cl}_{2},-80{ }^{\circ} \mathrm{C}\right): \delta$ $44.6\left(\mathrm{~s}, \mathrm{PPh}_{3}\right) .{ }^{11} \mathrm{~B}\left\{{ }^{1} \mathrm{H}\right\}$ NMR $\left(160 \mathrm{MHz}, \mathrm{CD}_{2} \mathrm{Cl}_{2},-80{ }^{\circ} \mathrm{C}\right) \delta-8.5$ (br s, 9-BBN). Anal. Calculated (found): C, 68.32 (68.63); H, 5.36 (5.55); N, 8.66 (8.70).

HRu(CH2 BCatMepi) $\mathbf{P P h}_{3}$ (3). $\mathrm{C}_{6} \mathrm{H}_{6}(10 \mathrm{~mL})$ was added to a $20 \mathrm{~mL}$ vial containing $\left[\mathrm{Ru}\left(\mathrm{CH}_{2} \mathrm{Mepi}\right) \mathrm{PPh}_{3}\right]_{2}(94.2 \mathrm{mg}, 0.0684 \mathrm{mmol}), \mathrm{HBCat}(32.8 \mathrm{mg}, 0.274 \mathrm{mmol})$, and a stir bar. The reaction solution was allowed to stir at $80{ }^{\circ} \mathrm{C}$ for $4 \mathrm{~h}$. After the solution cooled to room temperature, the $\mathrm{C}_{6} \mathrm{H}_{6}$ solvent was removed under vacuum and the crude product was washed with $\mathrm{Et}_{2} \mathrm{O}(5 \mathrm{~mL})$ and pentane $(4 \times 20 \mathrm{~mL})$. Evaporation of the volatiles under vacuum afforded the product as a brown powder. Crystals were obtained from vapor diffusion of pentane into a $\mathrm{C}_{6} \mathrm{H}_{6}$ solution at room temperature. Yield: $79 \mathrm{mg}(71 \%) .{ }^{1} \mathrm{H} \mathrm{NMR}\left(500 \mathrm{MHz}, \mathrm{CD}_{2} \mathrm{Cl}_{2}\right): \delta 8.15$ $\left(\right.$ br d, $\left.J_{\mathrm{HH}}=6.5 \mathrm{~Hz}, 1 \mathrm{H}\right), 7.72\left(\mathrm{br} \mathrm{d}, J_{\mathrm{HH}}=6.5 \mathrm{~Hz}, 1 \mathrm{H}\right), 7.63\left(\mathrm{t}, J_{\mathrm{HH}}=7.0 \mathrm{~Hz}, 1 \mathrm{H}\right), 7.54\left(\mathrm{t}, J_{\mathrm{HH}}=\right.$ $7.3 \mathrm{~Hz}, 1 \mathrm{H}), 7.42\left(\mathrm{t}, J_{\mathrm{HH}}=7.5 \mathrm{~Hz}, 1 \mathrm{H}\right), 7.18\left(\mathrm{t}, J_{\mathrm{HH}}=7.5 \mathrm{~Hz}, 3 \mathrm{H}, \mathrm{PPh}_{3}\right), 7.12\left(\mathrm{br} \mathrm{d}, J_{\mathrm{HH}}=7.5\right.$ $\mathrm{Hz}, 1 \mathrm{H}), 7.03-6.94(\mathrm{~m}, 14 \mathrm{H}), 6.56\left(\mathrm{~d}, J_{\mathrm{HH}}=7.5 \mathrm{~Hz}, 2 \mathrm{H}\right), 6.49\left(\mathrm{t}, J_{\mathrm{HH}}=7.5 \mathrm{~Hz}, 1 \mathrm{H}\right), 6.23\left(\mathrm{t}, J_{\mathrm{HH}}\right.$ $=7.5 \mathrm{~Hz}, 1 \mathrm{H}), 6.10\left(\mathrm{~d}, J_{\mathrm{HH}}=7.5 \mathrm{~Hz}, 1 \mathrm{H}\right), 5.98\left(\mathrm{~d}, J_{\mathrm{HH}}=6.5 \mathrm{~Hz}, 1 \mathrm{H}\right), 3.51\left(\mathrm{~d}, J_{\mathrm{HH}}=18.5 \mathrm{~Hz}\right.$, $1 \mathrm{H}), 2.66\left(\mathrm{dd}, J_{\mathrm{HH}}=18.5,4.8 \mathrm{~Hz}, 1 \mathrm{H}\right), 2.22$ (s, 3H, Me), -8.83 (br s, $1 \mathrm{H}$, hydride). ${ }^{13} \mathrm{C}\left\{{ }^{1} \mathrm{H}\right\}(176$ 
$\left.\mathrm{MHz}, \mathrm{CD}_{2} \mathrm{Cl}_{2}\right): \delta 167.38,164.38,152.53,151.55,148.37,135.35,134.88,133.81\left(\mathrm{~d}, J_{\mathrm{CP}}=9.3\right.$ $\mathrm{Hz}), 132.92,129.74,127.81\left(\mathrm{~d}, J_{\mathrm{CP}}=8.3 \mathrm{~Hz}\right), 125.93(\mathrm{br}), 121.78(\mathrm{br}), 120.14,119.43(\mathrm{br})$, 118.59 (br), 112.81, 110.71, 108.99, 33.17, 31.02. ${ }^{31} \mathrm{P}\left\{{ }^{1} \mathrm{H}\right\}$ NMR $\left(202 \mathrm{MHz}, \mathrm{CD}_{2} \mathrm{Cl}_{2}\right): \delta 79.9$ (s, $\left.\mathrm{PPh}_{3}\right) .{ }^{11} \mathrm{~B}\left\{{ }^{1} \mathrm{H}\right\}$ NMR $\left(160 \mathrm{MHz}, \mathrm{CD}_{2} \mathrm{Cl}_{2}\right) \delta 14.6$ (br s, BCat). Anal. Calculated (found): $\mathrm{C}, 65.35$ (65.16); H, 4.36 (4.13); N, 8.66 (8.46).

$\mathbf{R u}\left(\mathbf{C B P i n} \mathbf{M}_{2} \mathbf{M e p i}\right) \mathbf{P P h}_{3}$ (4). HBPin $(14.2 \mu \mathrm{L}, 0.0981 \mathrm{mmol})$ was added to a $20 \mathrm{~mL}$ vial containing a $\mathrm{C}_{6} \mathrm{H}_{6}(10 \mathrm{~mL})$ solution of $\left[\mathrm{Ru}\left(\mathrm{CH}_{2} \mathrm{Mepi}\right) \mathrm{PPh}_{3}\right]_{2}(30.7 \mathrm{mg}, 0.0223 \mathrm{mmol})$ and a stir bar. The reaction solution was allowed to stir at $80{ }^{\circ} \mathrm{C}$ for $20 \mathrm{~h}$. After the solution cooled to room temperature, the $\mathrm{C}_{6} \mathrm{H}_{6}$ solvent was removed under vacuum and the crude product was washed with pentane $(4 \times 10 \mathrm{~mL})$. The crude product was dissolved in minimum DCM and layered with pentane. After $24 \mathrm{~h}$ at $-35{ }^{\circ} \mathrm{C}$, the precipitates were collected and washed with cold pentane. Evaporation of the volatiles under vacuum afforded the product as a brown solid. Crystals were obtained from evaporation of a $\mathrm{C}_{6} \mathrm{H}_{6}$ solution at room temperature. Yield: $29 \mathrm{mg}(69 \%) .{ }^{1} \mathrm{H}$ NMR (500 MHz, $\left.\mathrm{C}_{6} \mathrm{D}_{6}\right): \delta 8.30\left(\mathrm{~d}, J_{\mathrm{HH}}=7.5 \mathrm{~Hz}, 1 \mathrm{H}\right), 8.15\left(\mathrm{~d}, J_{\mathrm{HH}}=7.0 \mathrm{~Hz}, 1 \mathrm{H}\right), 7.87\left(\mathrm{~d}, J_{\mathrm{HH}}=\right.$ $8.0 \mathrm{~Hz}, 1 \mathrm{H}), 7.37\left(\mathrm{t}, J_{\mathrm{HH}}=7.8 \mathrm{~Hz}, 1 \mathrm{H}\right), 7.30-7.27(\mathrm{~m}, 2 \mathrm{H}), 6.86-6.84(\mathrm{~m}, 9 \mathrm{H}), 6.45\left(\mathrm{~d}, J_{\mathrm{HH}}=\right.$ $7.5 \mathrm{~Hz}, 1 \mathrm{H}$ ), 2.60 (s, 3H, Me), 1.33 (s, 6H, BPin), 1.29 (s, 6H, BPin), 1.33 (s, 6H, BPin), 0.44 (br s, 6H, BPin), 0.33 (s, 6H, BPin). ${ }^{13} \mathrm{C}\left\{{ }^{1} \mathrm{H}\right\}\left(176 \mathrm{MHz}, \mathrm{CD}_{2} \mathrm{Cl}_{2}\right): \delta 175.26,165.80,158.26,153.17$, $152.29,150.76,142.62,141.25,134.89,134.57,134.33,133.68$ (d, $\left.J_{\mathrm{CP}}=8.4 \mathrm{~Hz}\right), 128.90$, $128.75,127.63\left(\mathrm{~d}, J_{\mathrm{CP}}=8.4 \mathrm{~Hz}\right), 126.21,120.25,119.47,116.62,116.45,81.68,31.69,26.19$ (br), 24.25. ${ }^{31} \mathrm{P}\left\{{ }^{1} \mathrm{H}\right\}$ NMR (162 MHz, $\left.\mathrm{C}_{6} \mathrm{D}_{6}\right): \delta 73.8\left(\mathrm{~s}, \mathrm{PPh}_{3}\right) .{ }^{11} \mathrm{~B}\left\{{ }^{1} \mathrm{H}\right\} \mathrm{NMR}\left(160 \mathrm{MHz}, \mathrm{C}_{6} \mathrm{D}_{6}\right) \delta$ 28.1 (br s, BPin). Anal. Calculated (found): C, 63.84 (63.70); H, 5.57 (5.76); N, 7.45 (7.54).

HRu( $\mathbf{C H}_{2}$ 9BBNMepi)(PPh $)$ CO (6). A J. Young tube containing a $\mathrm{C}_{6} \mathrm{H}_{6}$ solution of 3 ( $8.8 \mathrm{mg}, 0.0109 \mathrm{mmol}$ ) was charged with $\mathrm{H}_{2}(15 \mathrm{psig})$ and $\mathrm{CO}(30 \mathrm{psig})$. After $1 \mathrm{~h}$ at room temperature, the $\mathrm{C}_{6} \mathrm{H}_{6}$ solvent was removed under vacuum and the crude product was extracted with pentane. After $48 \mathrm{~h}$ at $-35{ }^{\circ} \mathrm{C}$, the precipitates were collected and dried under vacuum to afford the product as orange needles. Yield: $5 \mathrm{mg}(55 \%)$. ${ }^{1} \mathrm{H}$ NMR (700 $\left.\mathrm{MHz} \mathrm{C}_{6} \mathrm{D}_{6}\right): \delta 7.70(\mathrm{~d}$, $\left.J_{\mathrm{HH}}=7.0 \mathrm{~Hz}, 1 \mathrm{H}\right), 7.61\left(\mathrm{t}, J_{\mathrm{HH}}=7.4 \mathrm{~Hz}, 1 \mathrm{H}\right), 7.56\left(\mathrm{t}, J_{\mathrm{HH}}=7.4 \mathrm{~Hz}, 1 \mathrm{H}\right), 7.47\left(\mathrm{~d}, J_{\mathrm{HH}}=7.7 \mathrm{~Hz}\right.$, 2H), $7.41\left(\mathrm{t}, J_{\mathrm{HH}}=7.0 \mathrm{~Hz}, 1 \mathrm{H}\right), 7.34\left(\mathrm{t}, J_{\mathrm{HH}}=7.0 \mathrm{~Hz}, 1 \mathrm{H}\right), 7.14-7.09(\mathrm{~m}, 4 \mathrm{H}), 6.97\left(\mathrm{t}, J_{\mathrm{HH}}=7.0\right.$ $\left.\mathrm{Hz}, 6 \mathrm{H}, \mathrm{PPh}_{3}\right), 6.91-6.85(\mathrm{~m}, 8 \mathrm{H}), 3.64\left(\mathrm{~d}, J_{\mathrm{HH}}=16.1 \mathrm{~Hz}, 1 \mathrm{H}\right), 2.64\left(\mathrm{~d}, J_{\mathrm{HH}}=19.6 \mathrm{~Hz}, 1 \mathrm{H}\right), 2.23$ (s, 3H, Me), 1.81 (br s, 1H), 1.57 (br s, 2H), 1.45 (br s, 1H), 1.34-1.23 (br m, 3H), 1.09 (br s, 
3H), 1.01 (br s, 1H), 0.93 (br s, 1H), $0.23($ br s, $1 \mathrm{H}),-0.37$ (br s, 1H), -10.38 (br d, $J_{\mathrm{HP}}=91.7$ $\mathrm{Hz}, 1 \mathrm{H}$, hydride). ${ }^{13} \mathrm{C}\left\{{ }^{1} \mathrm{H}\right\} \quad\left(176 \mathrm{MHz}, \mathrm{CD}_{2} \mathrm{Cl}_{2}\right): \delta 160.89,160.74,156.20,140.53,137.17$, $136.91,133.83\left(\mathrm{~d}, J_{\mathrm{CP}}=9.2 \mathrm{~Hz}\right), 133.05,130.12,130.02,129.58,129.15,128.70,128.15\left(\mathrm{~d}, J_{\mathrm{CP}}\right.$ $=8.1 \mathrm{~Hz}), 127.43,126.47,121.70,121.58,121.48,120.41,120.13,34.04,33.83,32.61,31.97$, 26.22, 25.64. ${ }^{31} \mathrm{P}\left\{{ }^{1} \mathrm{H}\right\}$ NMR $\left(202 \mathrm{MHz}, \mathrm{C}_{6} \mathrm{D}_{6}\right): \delta 38.6\left(\mathrm{~s}, \mathrm{PPh}_{3}\right) .{ }^{11} \mathrm{~B}\left\{{ }^{1} \mathrm{H}\right\} \mathrm{NMR}\left(160 \mathrm{MHz}, \mathrm{C}_{6} \mathrm{D}_{6}\right)$ $\delta-6.5$ (br s, 9-BBN). IR (ATR, $\mathrm{cm}^{-1}$ ): 3059, 2908, 2832, 1935, 1809, 1571, 1515, 1432, 1388, 1318, 1283, 1249, 1186, 1111, 1090, 989, 973, 907, 888, 859, 821, 793, 774, 740, 694, 672. Anal. Calculated (found): C, 67.30 (66.88); H, 5.41 (5.33); N, 8.35 (7.85). 


\section{Solution Characterization of $\mathbf{5}$ :}

Complementary to the solid-state characterization, the solution structure of $\mathbf{5}$ was investigated using variable temperature NMR spectroscopy. At $25{ }^{\circ} \mathrm{C}$, the ${ }^{1} \mathrm{H}$ NMR spectrum exhibited broad signals in the alkyl region and a broad signal at $-3.5 \mathrm{ppm}$, and the ${ }^{11} \mathrm{~B}\left\{{ }^{1} \mathrm{H}\right\} \mathrm{NMR}$ spectrum was featureless. However, upon cooling a $\mathrm{CD}_{2} \mathrm{Cl}_{2}$ solution of 5 to $-80{ }^{\circ} \mathrm{C}$, the ${ }^{1} \mathrm{H}$ signals sharpened and a broad signal appeared at $-8.5 \mathrm{ppm}$ in the ${ }^{11} \mathrm{~B}\left\{{ }^{1} \mathrm{H}\right\}$ NMR spectrum, indicative of a fluxional structure at room temperature. Moreover, in the ${ }^{1} \mathrm{H}$ NMR spectrum at $-80{ }^{\circ} \mathrm{C}$, the signal at -3.87 ppm appeared as a well-resolved doublet with a coupling constant of $16.5 \mathrm{~Hz}$ that appeared concomitantly with a doublet at $2.06 \mathrm{ppm}$ with the same coupling constant and a $T_{1}(\mathrm{~min})$ of 211 $\mathrm{ms}\left(-40{ }^{\circ} \mathrm{C}, 500 \mathrm{MHz}\right)$ (Figure S5). This observation is consistent with an agostic interaction of a geminal $-\mathrm{CH}_{2}$ group with $\mathrm{Ru} .^{6}$ Thus, we propose two coordination modes of the 9-BBN motif to the $\mathrm{Ru}$ center $\left(\mathrm{Ru}-\left(\eta^{2}-\mathrm{B}-\mathrm{C}\right)\right.$ and a $\mathrm{C}-\mathrm{H}$ agostic interaction, Figure S5).
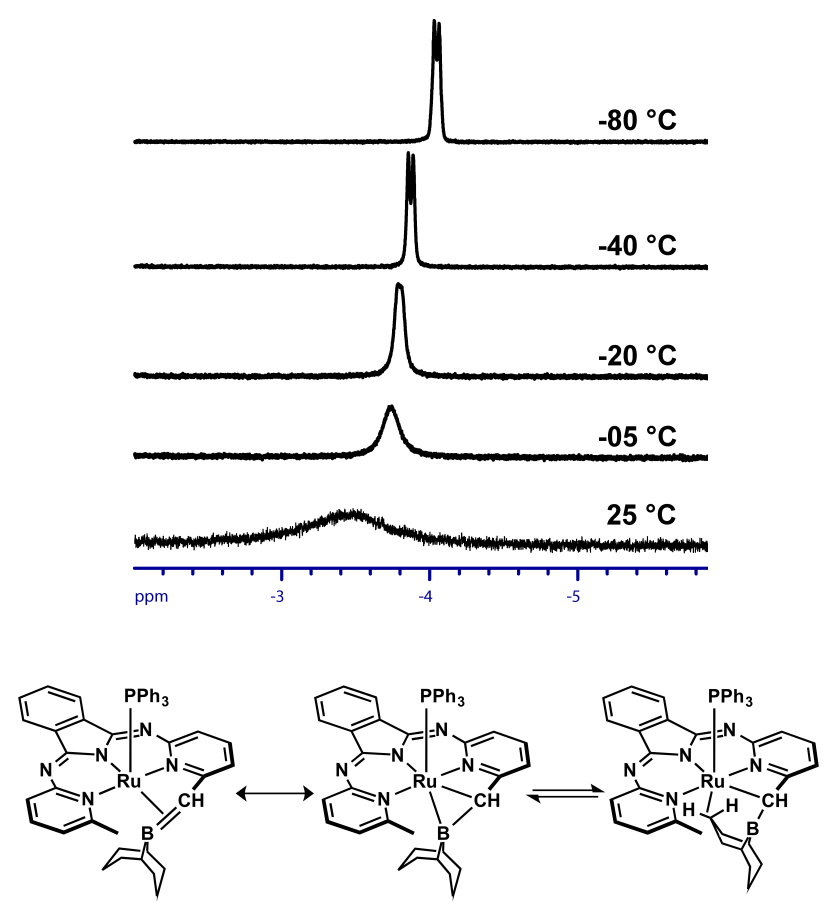

Figure S5. (Top) Dynamic coordination of the 9-BBN group in complex 3 investigated using variable temperature ${ }^{1} \mathrm{H}$ NMR spectroscopy $\left(500 \mathrm{MHz}, \mathrm{CD}_{2} \mathrm{Cl}_{2}\right.$ ). (Bottom) Limiting resonance description for $\mathbf{5}$ and solution equilibrium process. 


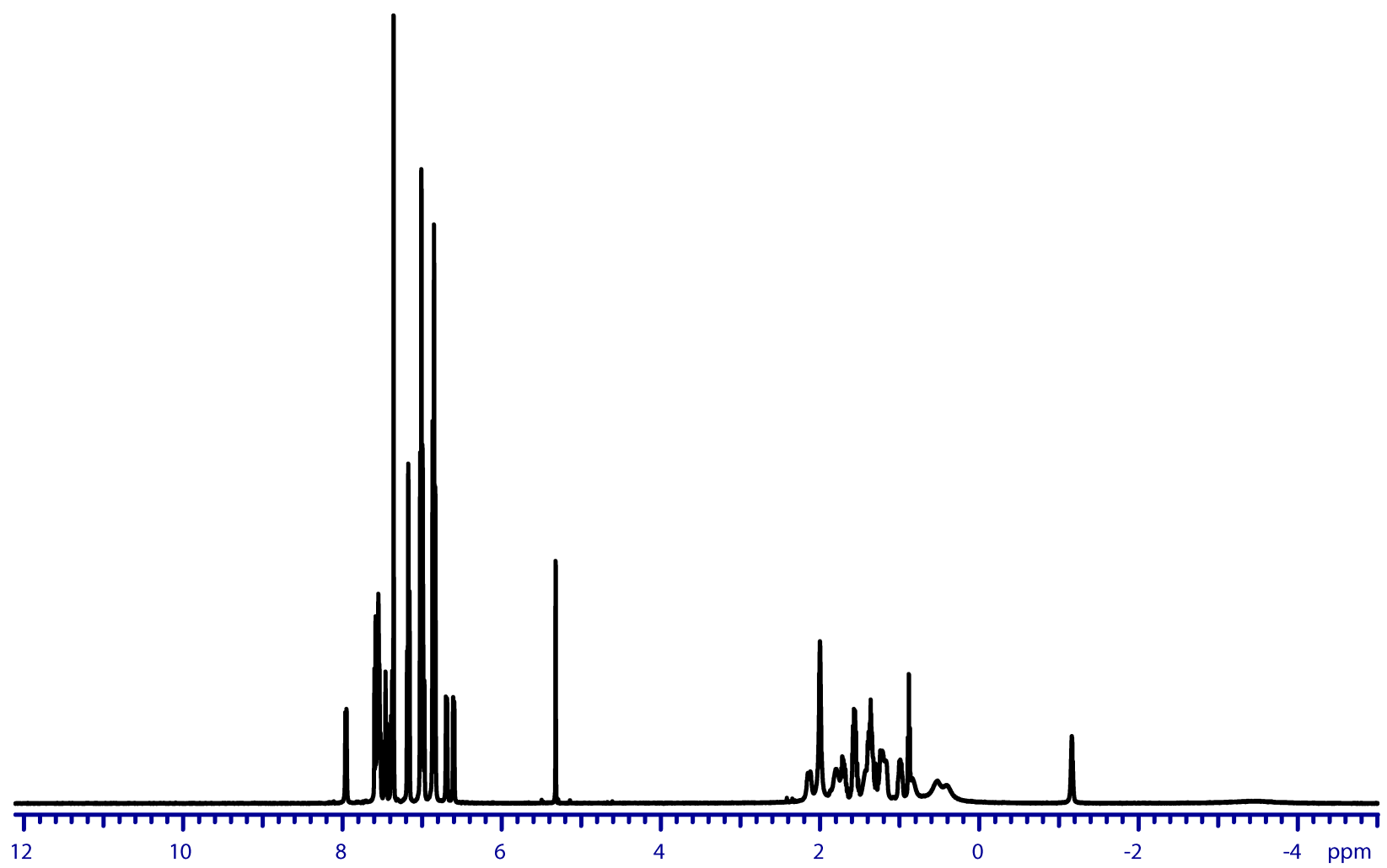

Figure S6. ${ }^{1} \mathrm{H} \mathrm{NMR}\left(500 \mathrm{MHz}, \mathrm{CD}_{2} \mathrm{Cl}_{2}\right)$ spectrum of $\mathrm{Ru}(\mathrm{CH} 9 \mathrm{BBNMepi}) \mathrm{PPh}_{3}$.

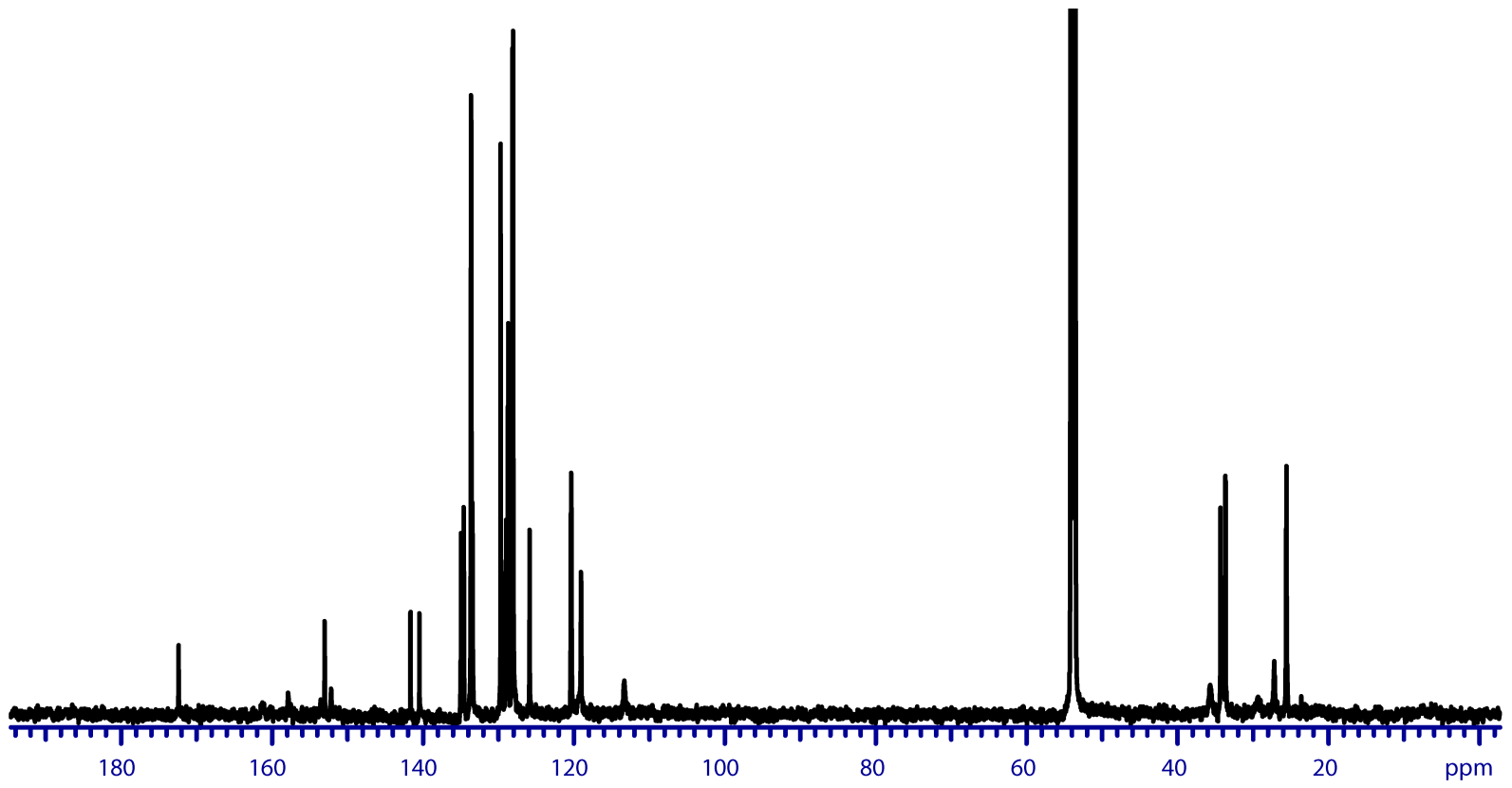

Figure S7. ${ }^{13} \mathrm{C}\left\{{ }^{1} \mathrm{H}\right\} \mathrm{NMR}\left(176 \mathrm{MHz}, \mathrm{CD}_{2} \mathrm{Cl}_{2}\right)$ spectrum of $\mathrm{Ru}(\mathrm{CH} 9 \mathrm{BBNMepi}) \mathrm{PPh}_{3}$. 


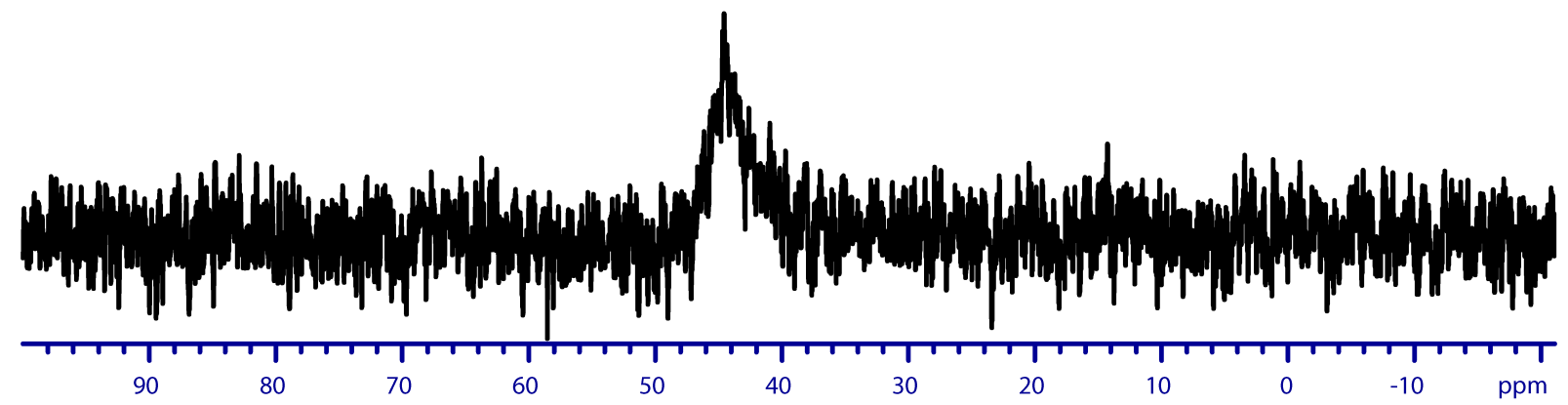

Figure S8. ${ }^{31} \mathrm{P}\left\{{ }^{1} \mathrm{H}\right\}$ NMR (162 MHz, $\left.\mathrm{C}_{6} \mathrm{D}_{6}\right)$ spectrum of $\mathrm{Ru}(\mathrm{CH} 9 \mathrm{BBNMepi}) \mathrm{PPh}_{3}$.

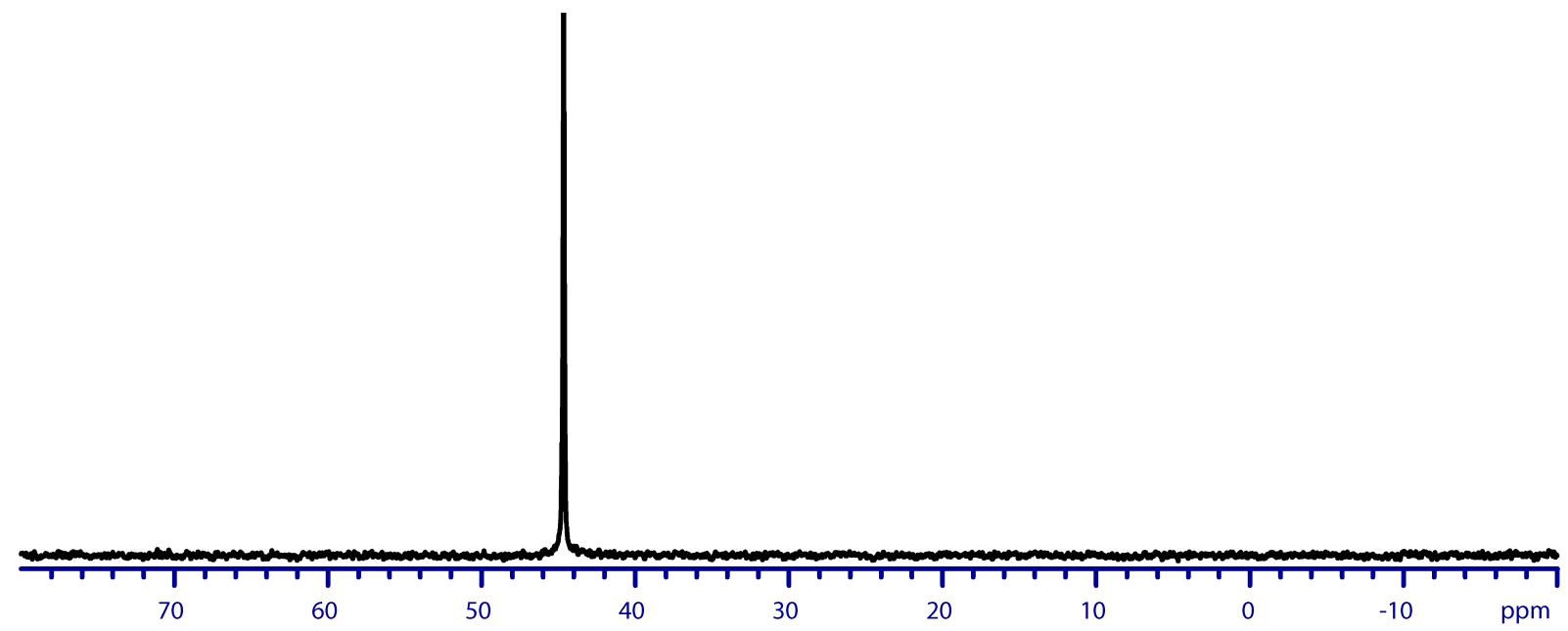

Figure S9. ${ }^{31} \mathrm{P}\left\{{ }^{1} \mathrm{H}\right\} \mathrm{NMR}\left(162 \mathrm{MHz}, \mathrm{CD}_{2} \mathrm{Cl}_{2},-80{ }^{\circ} \mathrm{C}\right)$ spectrum of $\mathrm{Ru}(\mathrm{CH} 9 \mathrm{BBNMepi}) \mathrm{PPh}_{3}$.

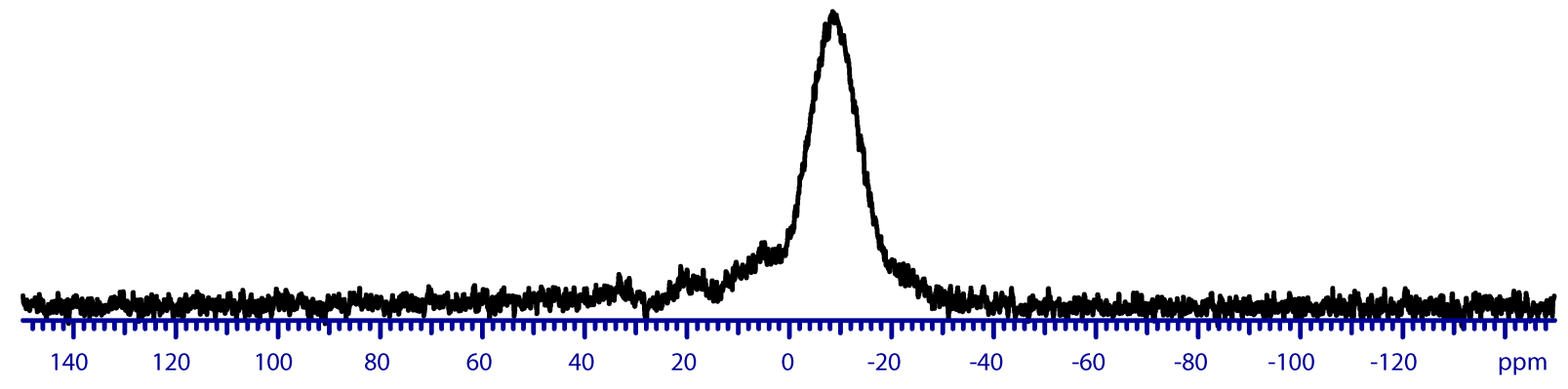

Figure S10. ${ }^{11} \mathrm{~B}\left\{{ }^{1} \mathrm{H}\right\}$ NMR $\left(160 \mathrm{MHz}, \mathrm{CD}_{2} \mathrm{Cl}_{2},-80{ }^{\circ} \mathrm{C}\right)$ spectrum of $\mathrm{Ru}(\mathrm{CH} 9 \mathrm{BBNMepi}) \mathrm{PPh}_{3}$. 


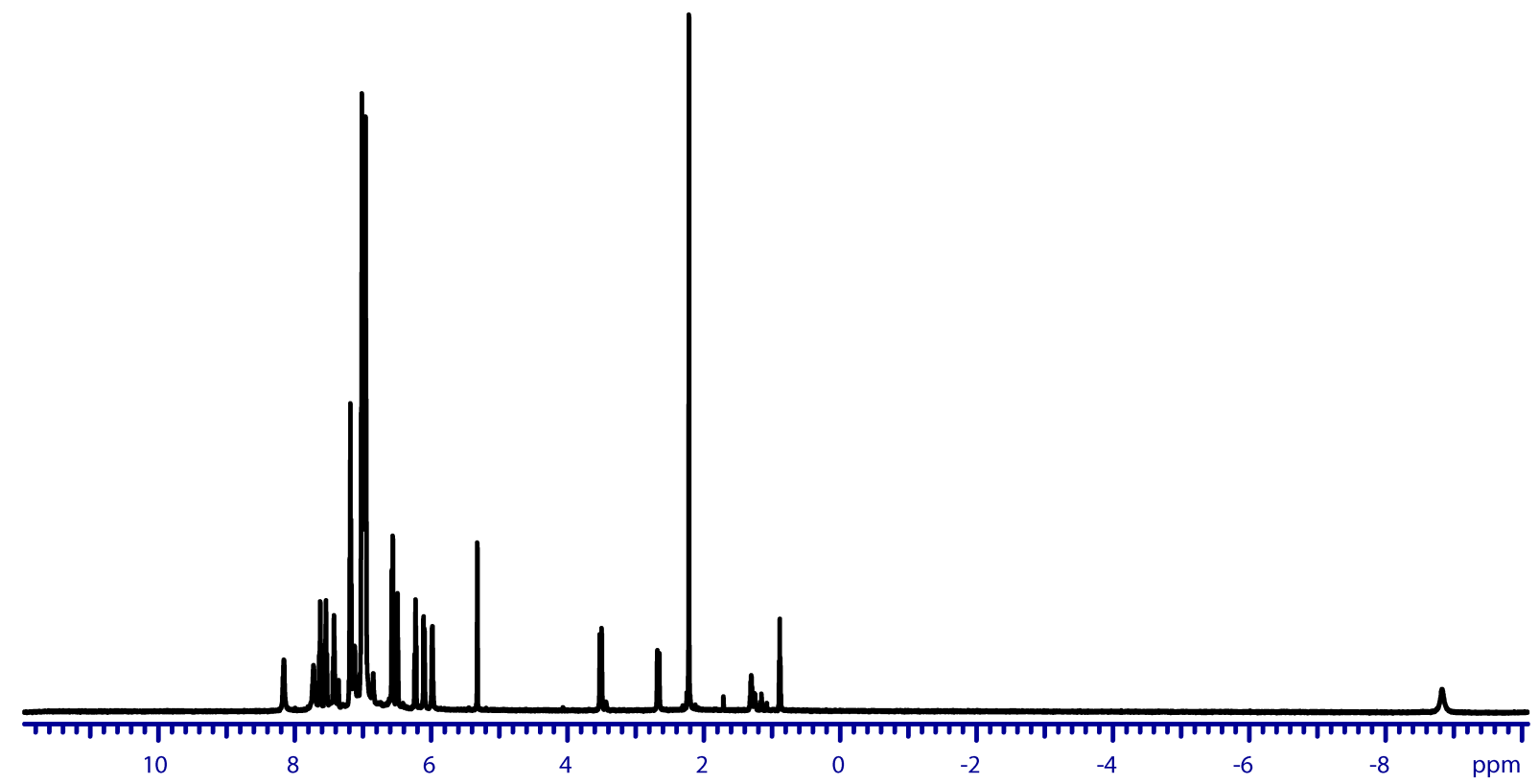

Figure S11. ${ }^{1} \mathrm{H} \mathrm{NMR}\left(500 \mathrm{MHz}, \mathrm{CD}_{2} \mathrm{Cl}_{2}\right)$ spectrum of $\mathrm{HRu}\left(\mathrm{CH}_{2} \mathrm{BCatMepi}\right) \mathrm{PPh}_{3}$.

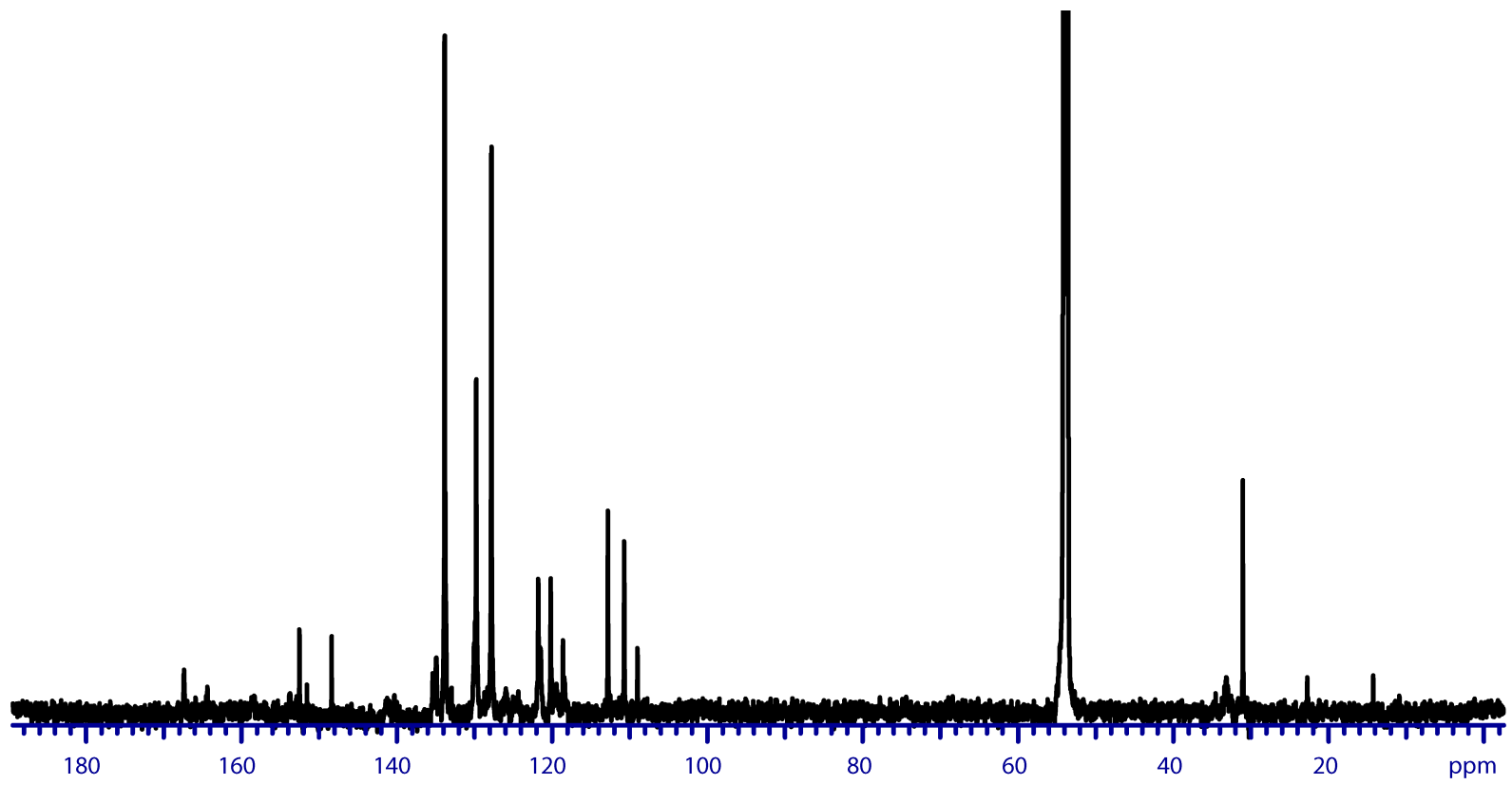

Figure S12. ${ }^{13} \mathrm{C}\left\{{ }^{1} \mathrm{H}\right\} \mathrm{NMR}\left(176 \mathrm{MHz}, \mathrm{CD}_{2} \mathrm{Cl}_{2}\right)$ spectrum of $\mathrm{HRu}\left(\mathrm{CH}_{2} \mathrm{BCatMepi}\right) \mathrm{PPh}_{3}$. 


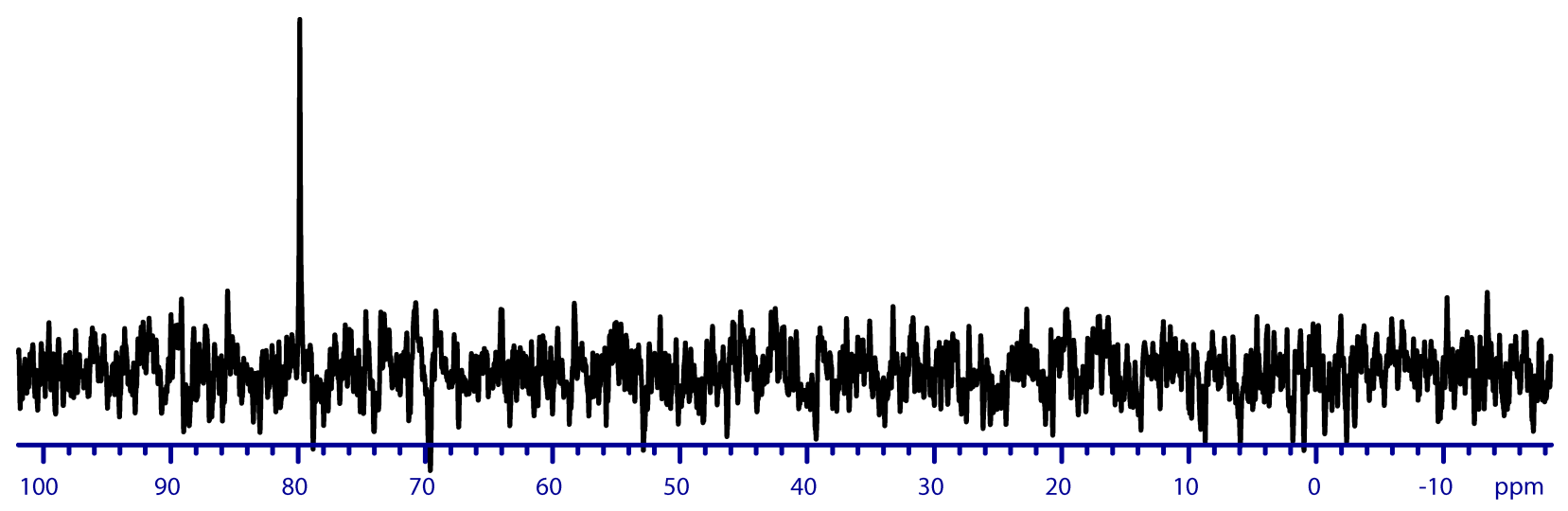

Figure S13. ${ }^{31} \mathrm{P}\left\{{ }^{1} \mathrm{H}\right\} \mathrm{NMR}\left(162 \mathrm{MHz}, \mathrm{C}_{6} \mathrm{D}_{6}\right)$ spectrum of $\mathrm{HRu}\left(\mathrm{CH}_{2} \mathrm{BCatMepi}\right) \mathrm{PPh}_{3}$.

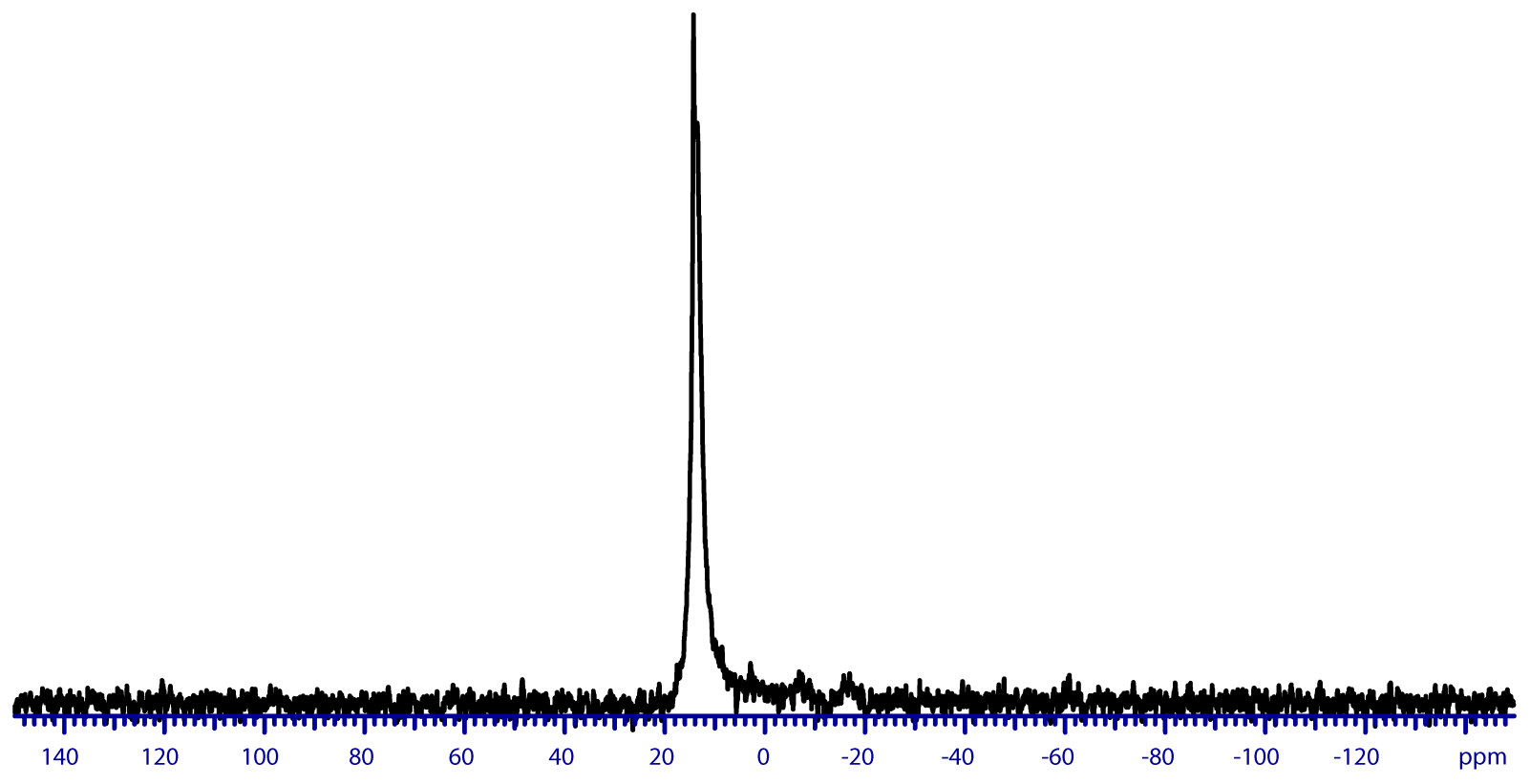

Figure S14. ${ }^{11} \mathrm{~B}\left\{{ }^{1} \mathrm{H}\right\} \mathrm{NMR}\left(160 \mathrm{MHz}, \mathrm{CD}_{2} \mathrm{Cl}_{2}\right)$ spectrum of $\mathrm{HRu}\left(\mathrm{CH}_{2} \mathrm{BCatMepi}\right) \mathrm{PPh}_{3}$. 


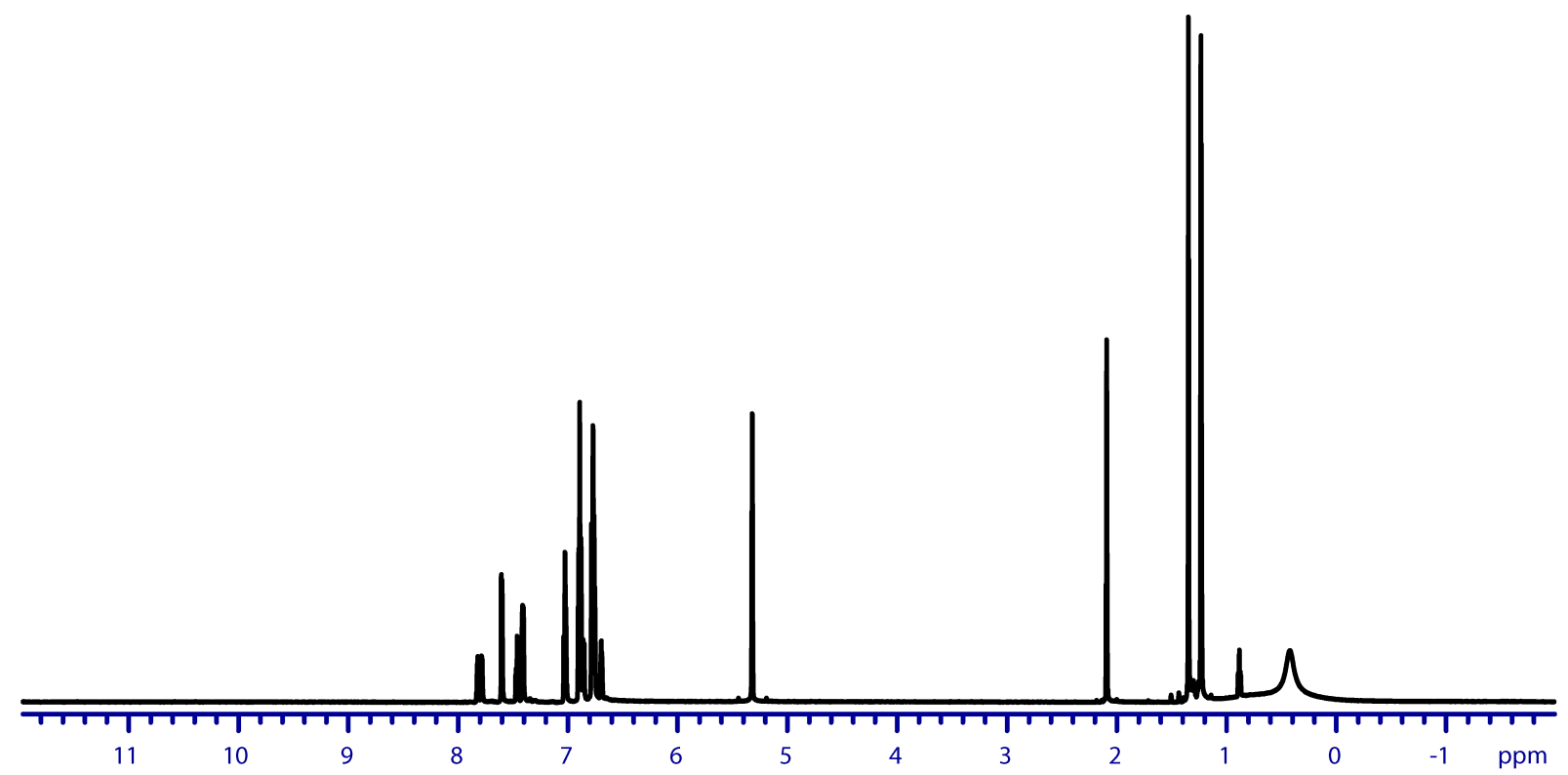

Figure S15. ${ }^{1} \mathrm{H} \mathrm{NMR}\left(700 \mathrm{MHz}, \mathrm{CD}_{2} \mathrm{Cl}_{2}\right)$ spectrum of $\mathrm{Ru}\left(\mathrm{CBPin}{ }_{2} \mathrm{Mepi}\right) \mathrm{PPh}_{3}$.

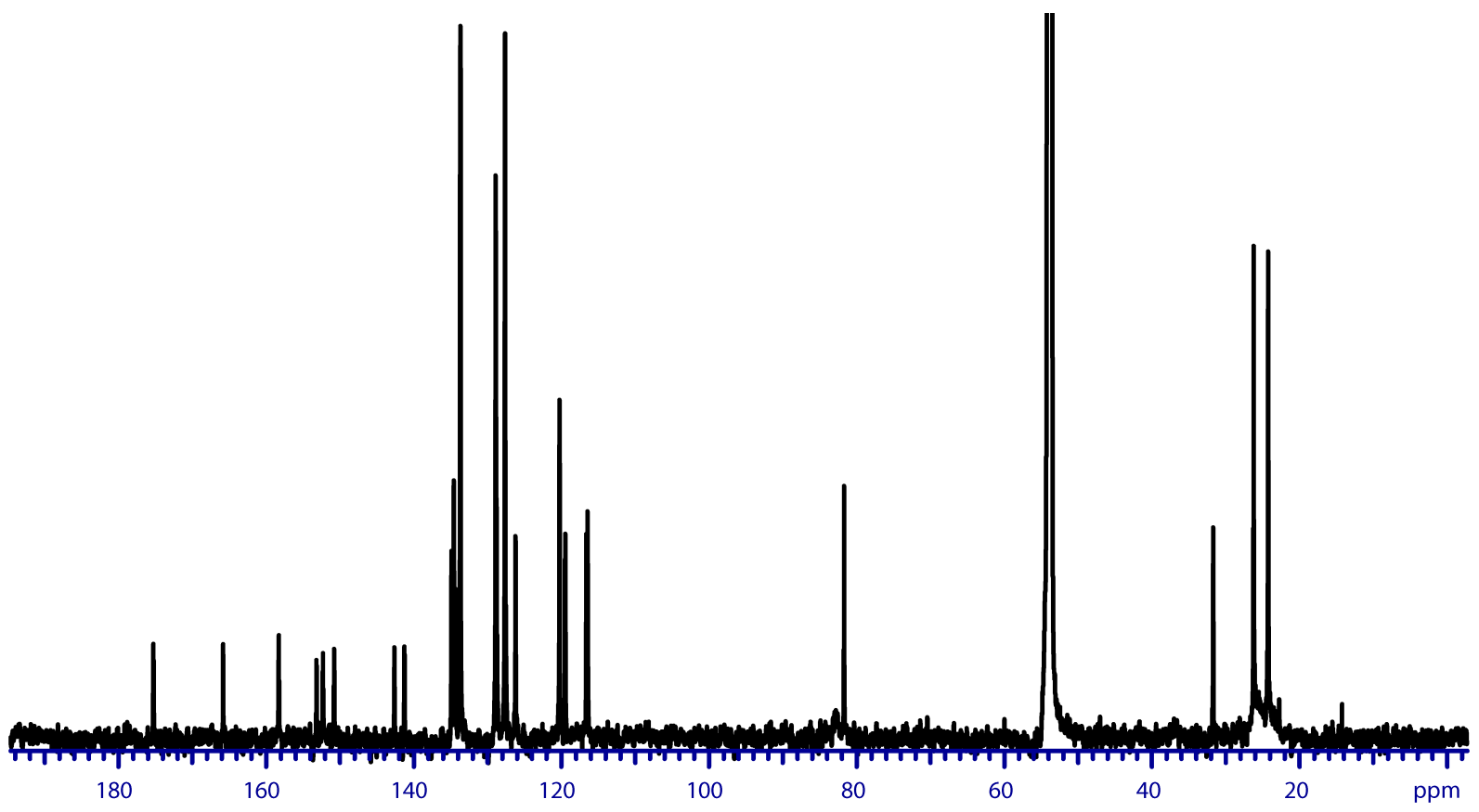

Figure S16. ${ }^{13} \mathrm{C}\left\{{ }^{1} \mathrm{H}\right\}$ NMR $\left(176 \mathrm{MHz}, \mathrm{CD}_{2} \mathrm{Cl}_{2}\right)$ spectrum of $\mathrm{Ru}\left(\mathrm{CBPin}{ }_{2} \mathrm{Mepi}\right) \mathrm{PPh}_{3}$. 


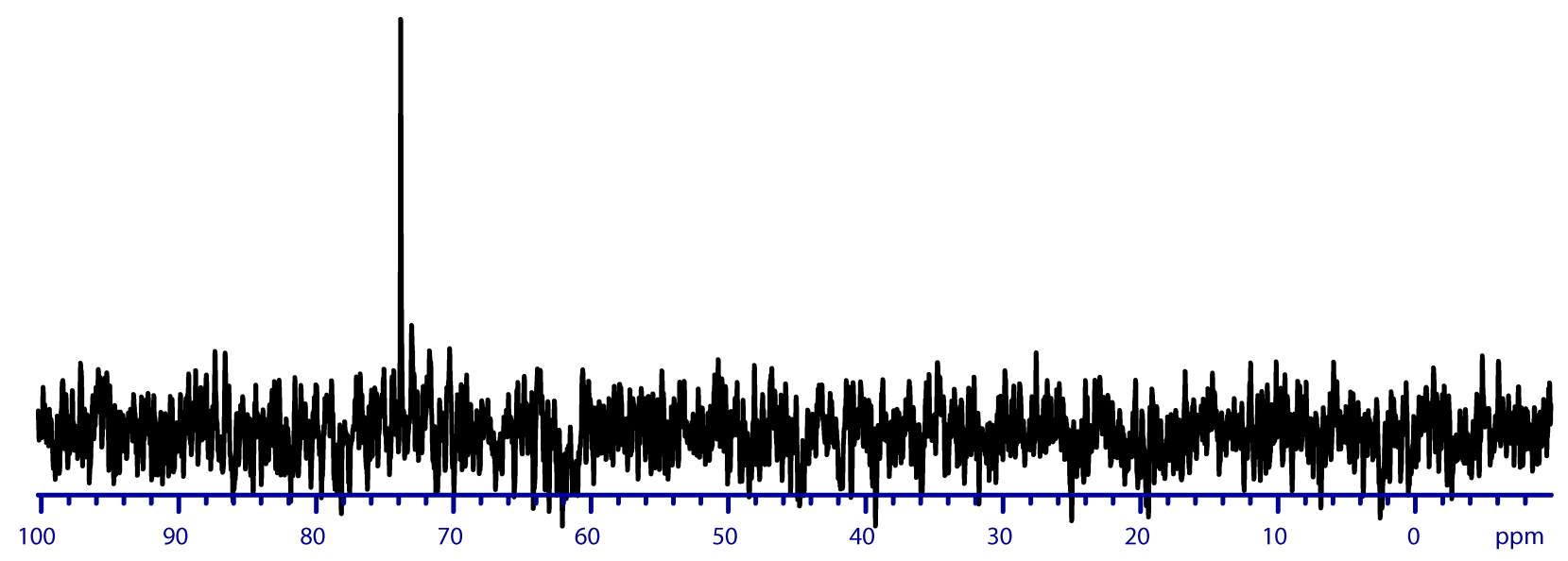

Figure S17. ${ }^{31} \mathrm{P}\left\{{ }^{1} \mathrm{H}\right\} \mathrm{NMR}\left(162 \mathrm{MHz}, \mathrm{C}_{6} \mathrm{D}_{6}\right)$ spectrum of $\mathrm{Ru}\left(\mathrm{CBPin}{ }_{2} \mathrm{Mepi}\right) \mathrm{PPh}_{3}$.

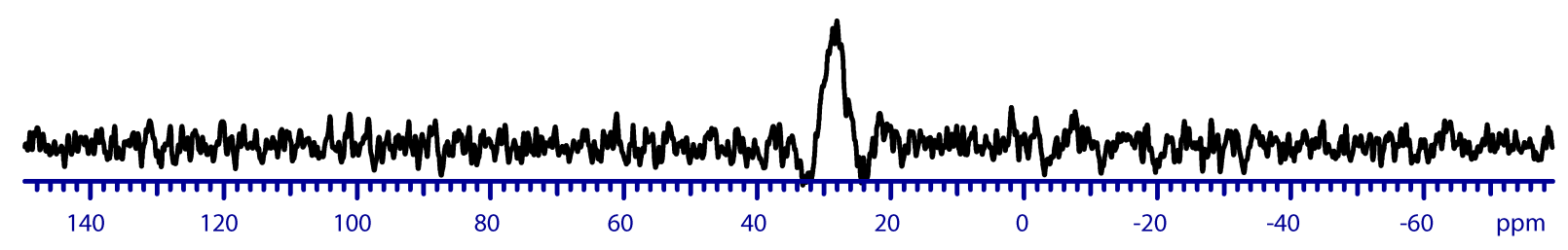

Figure S18. ${ }^{11} \mathrm{~B}\left\{{ }^{1} \mathrm{H}\right\} \mathrm{NMR}\left(160 \mathrm{MHz}, \mathrm{C}_{6} \mathrm{D}_{6}\right)$ spectrum of $\mathrm{Ru}\left(\mathrm{CBPin}{ }_{2} \mathrm{Mepi}\right) \mathrm{PPh}_{3}$. 


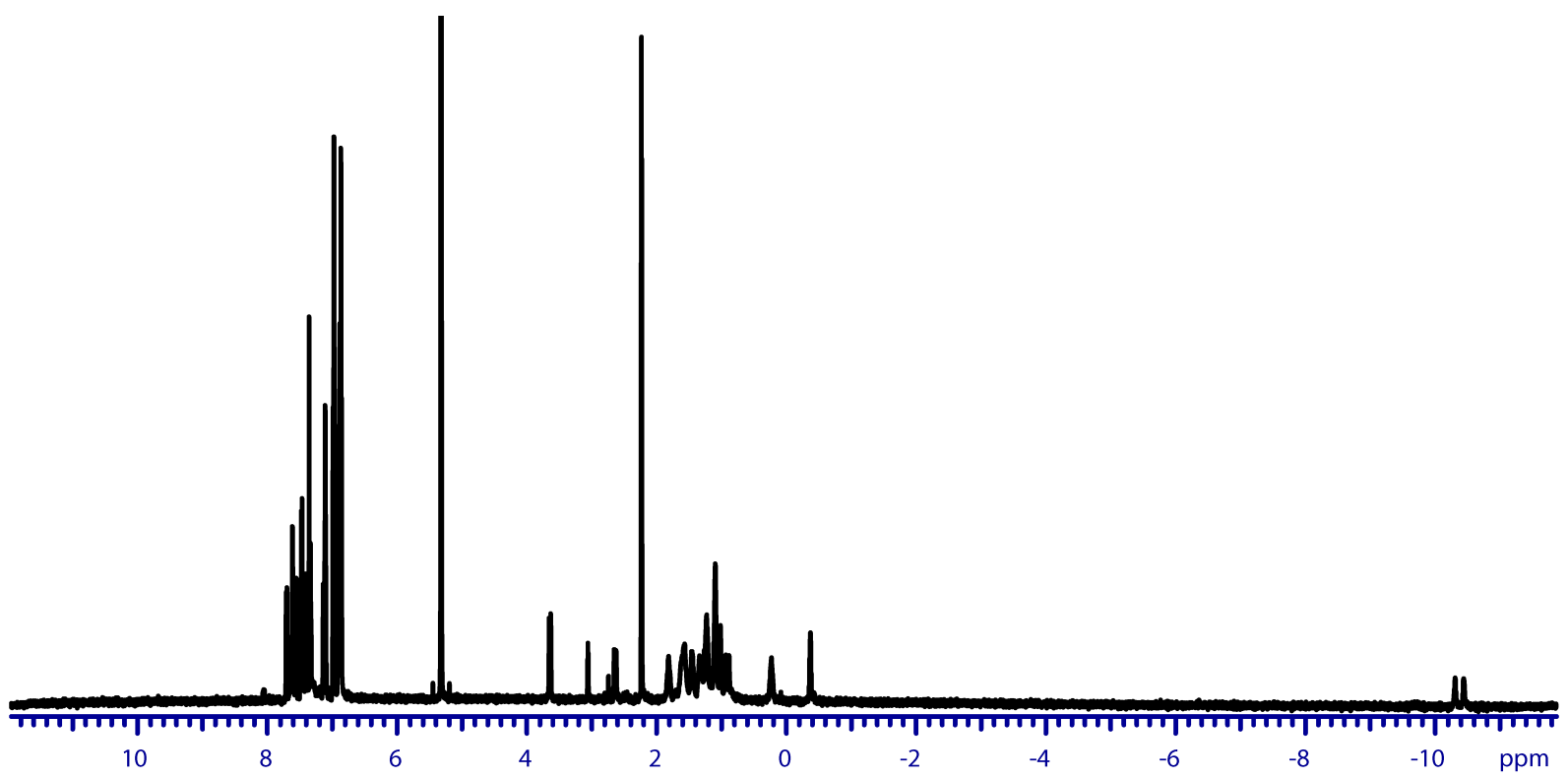

Figure S19. ${ }^{1} \mathrm{H} \mathrm{NMR}\left(700 \mathrm{MHz}, \mathrm{CD}_{2} \mathrm{Cl}_{2}\right)$ spectrum of $\mathrm{HRu}\left(\mathrm{CH}_{2} 9 \mathrm{BBNMepi}\right)\left(\mathrm{PPh}_{3}\right) \mathrm{CO}$.

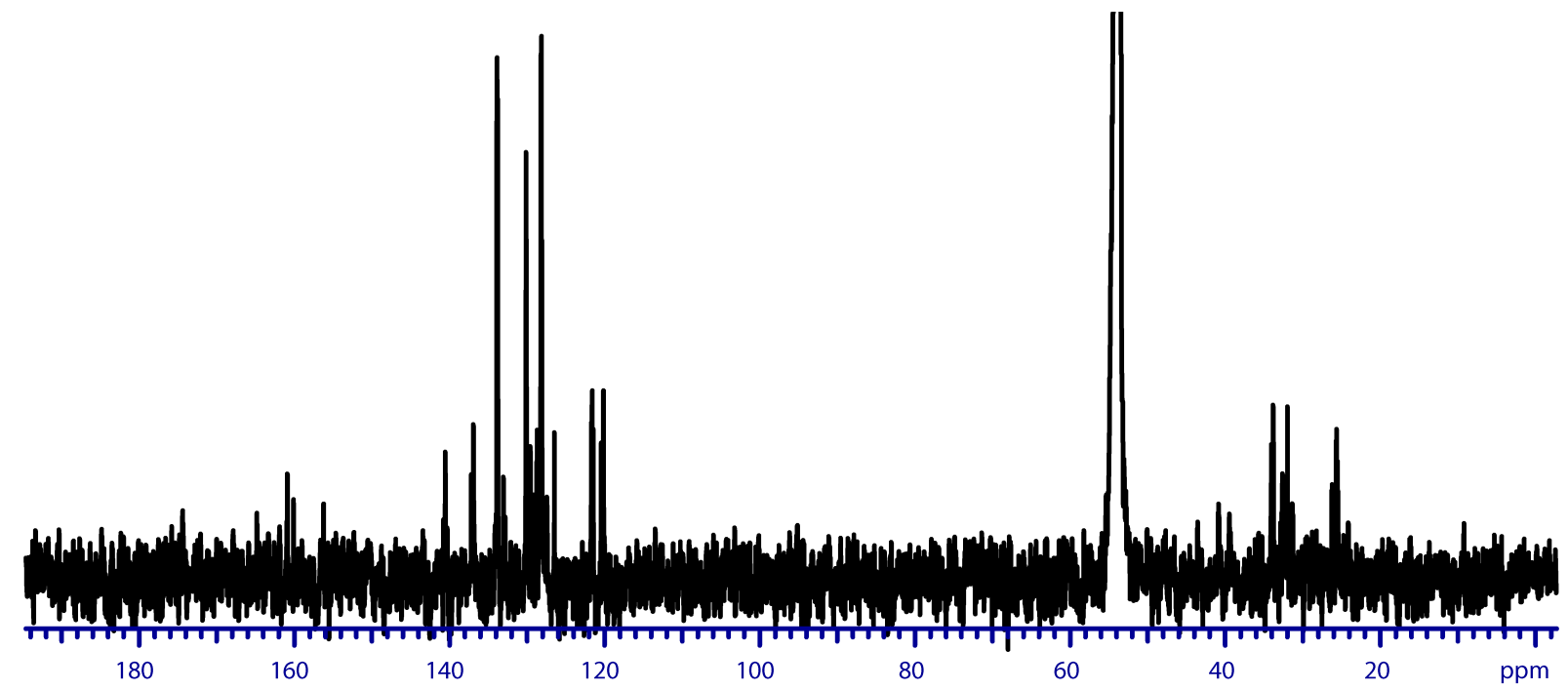

Figure S20. ${ }^{13} \mathrm{C}\left\{{ }^{1} \mathrm{H}\right\} \mathrm{NMR}\left(176 \mathrm{MHz}, \mathrm{CD}_{2} \mathrm{Cl}_{2}\right)$ spectrum of $\mathrm{HRu}\left(\mathrm{CH}_{2} 9 \mathrm{BBNMepi}\right)\left(\mathrm{PPh}_{3}\right) \mathrm{CO}$. 


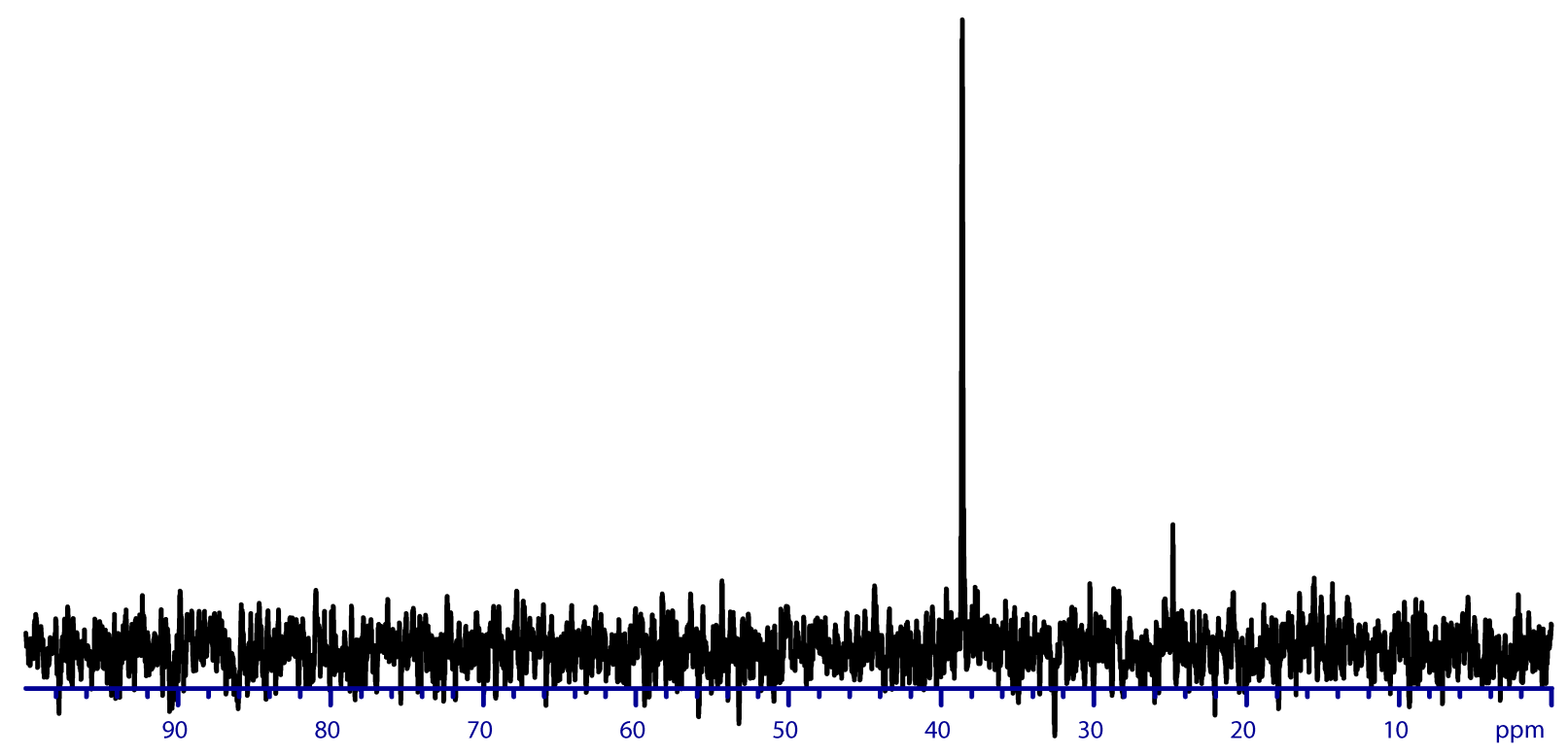

Figure S21. ${ }^{31} \mathrm{P}\left\{{ }^{1} \mathrm{H}\right\}$ NMR $\left(162 \mathrm{MHz}, \mathrm{C}_{6} \mathrm{D}_{6}\right)$ spectrum of $\mathrm{HRu}\left(\mathrm{CH}_{2} 9 \mathrm{BBNMepi}\right)\left(\mathrm{PPh}_{3}\right) \mathrm{CO}$.

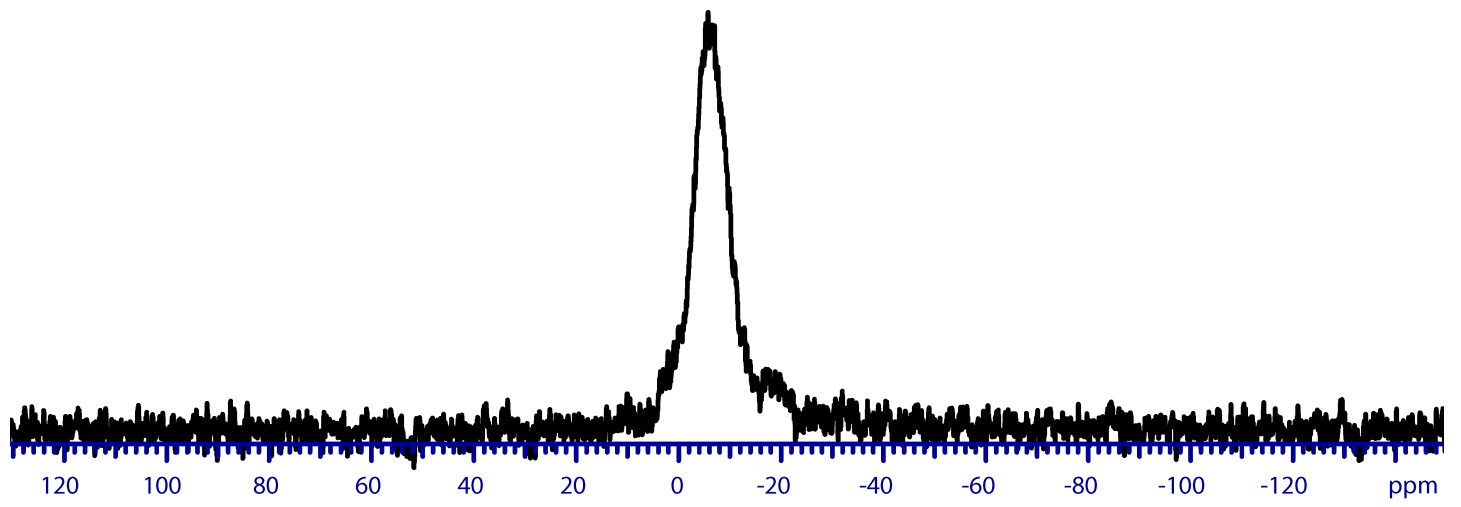

Figure S22. ${ }^{11} \mathrm{~B}\left\{{ }^{1} \mathrm{H}\right\} \mathrm{NMR}\left(160 \mathrm{MHz}, \mathrm{C}_{6} \mathrm{D}_{6}\right)$ spectrum of $\mathrm{HRu}\left(\mathrm{CH}_{2} 9 \mathrm{BBNMepi}\right)\left(\mathrm{PPh}_{3}\right) \mathrm{CO}$. 


\section{Competition Experiments between Alkyne and Alkene with $\mathrm{HRu}\left(\mathrm{CH}_{2} \mathrm{BCatMepi}\right) \mathrm{PPh}_{3}$}

To gain further insight into the origin of the preference for the alkyne over alkene, we evaluated stoichiometric competition experiments between 1-octyne and 1-octene. The addition of a $\mathrm{C}_{6} \mathrm{H}_{6}$ solution containing a mixture 1 equiv of 1-octyne and 1-octene to $\mathbf{3}$ resulted in the exclusive conversion to alkyne insertion products, as confirmed by identical ${ }^{31} \mathrm{P}$ and ${ }^{1} \mathrm{H}$ NMR spectra when 3 was allowed to react with 2 equiv of 1-octyne. Furthermore, the insertion reaction of alkyne was also maintained even when using 1000:1 mixtures of 1-octene to 1-octyne. In contrast, when the competition experiments were performed using 1 in the presence of 1000:1 of 1-octene to 1octyne, the ${ }^{31} \mathrm{P}$ NMR spectrum featured a broad peak at $-5.4 \mathrm{ppm}\left(\right.$ free $\left.\mathrm{PPh}_{3}\right)$ and the ${ }^{1} \mathrm{H}$ NMR spectrum showed broad peaks that were not resolved at low temperature. This is consistent with a very fast dynamic equilibrium process between hydride insertion into the alkene and alkyne.

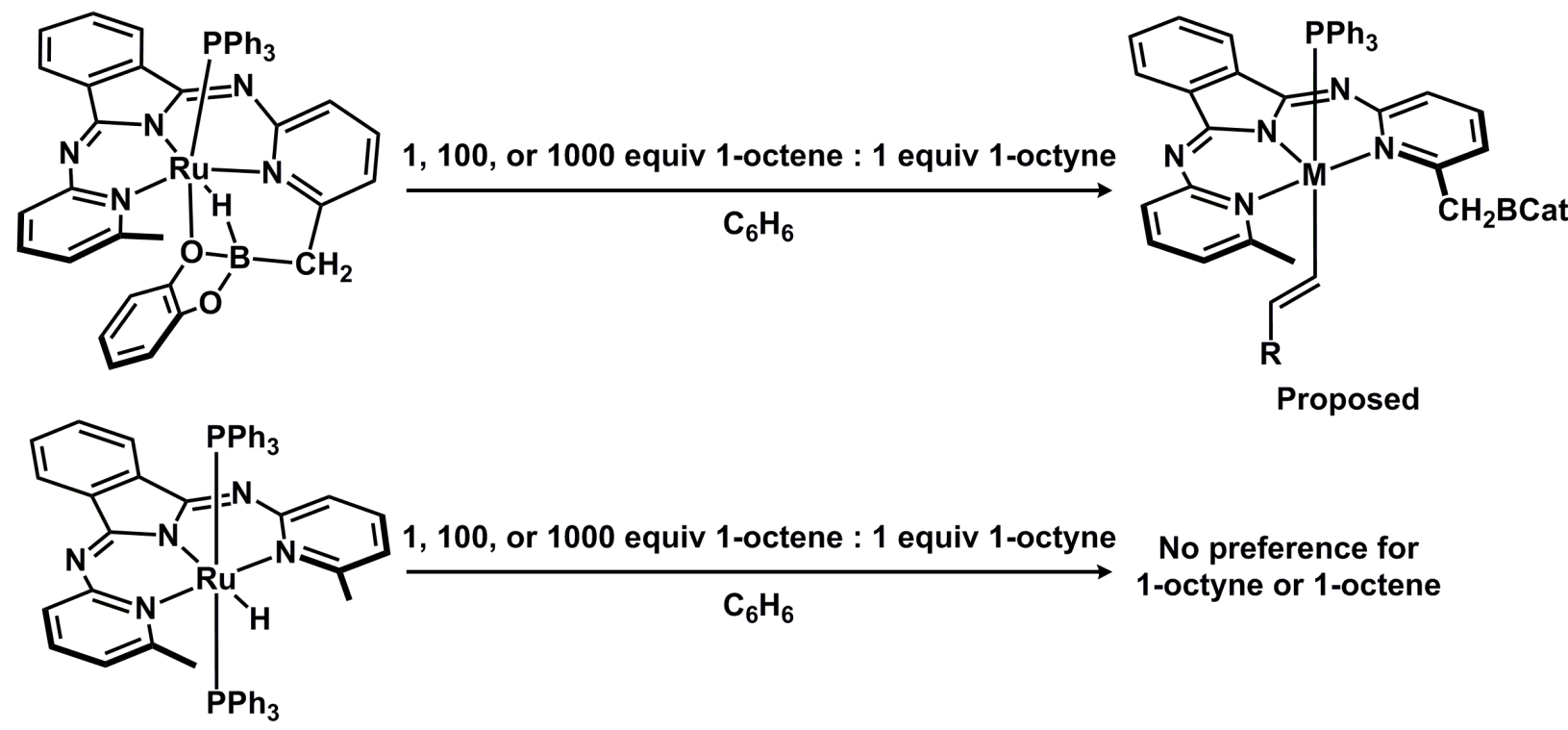




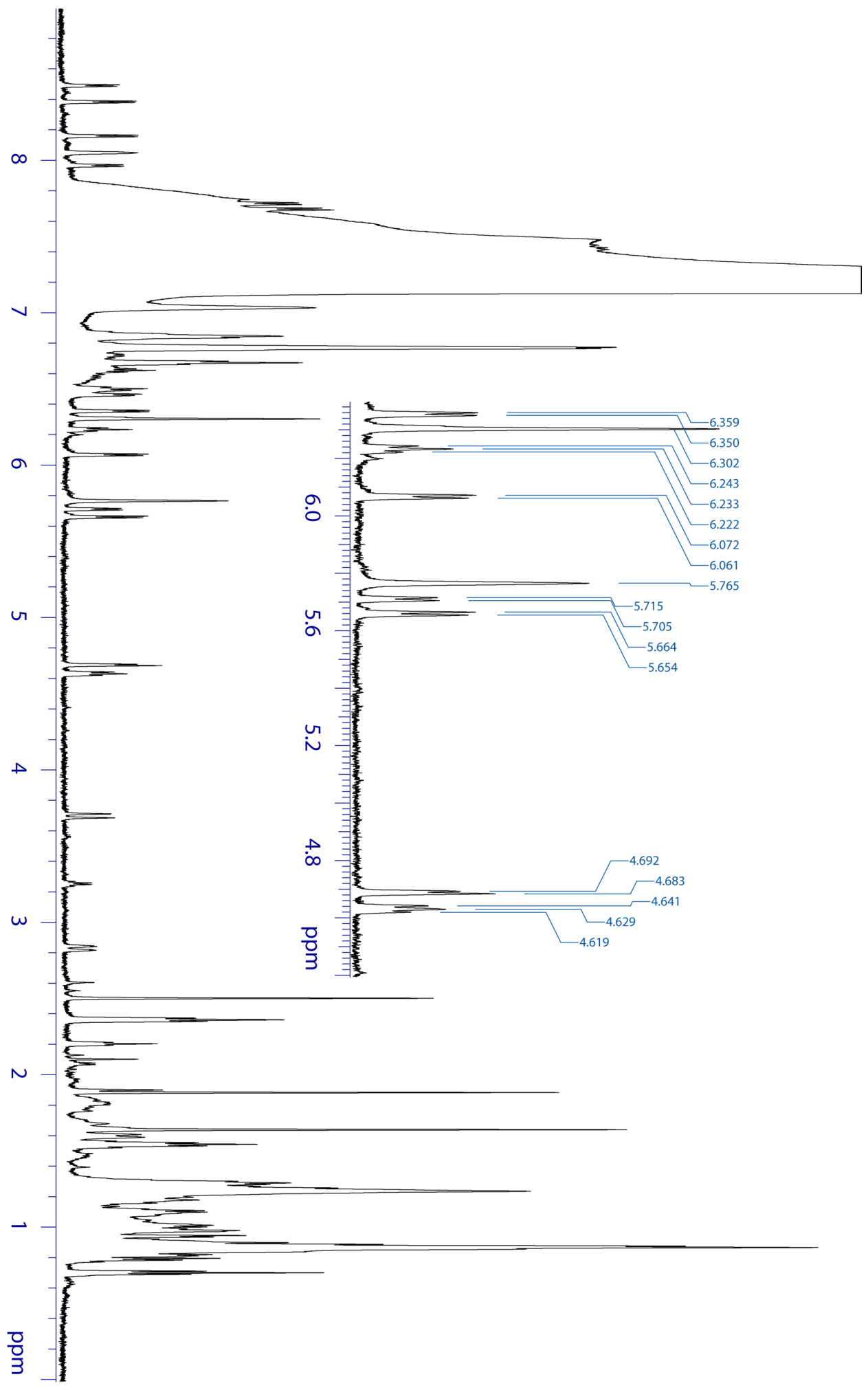

Figure S23. ${ }^{1} \mathrm{H}$ NMR $\left(700 \mathrm{MHz}, \mathrm{C}_{6} \mathrm{H}_{6}\right)$ spectrum of $\mathrm{HRu}\left(\mathrm{CH}_{2} \mathrm{BCatMepi}\right) \mathrm{PPh}_{3}$ and 2 equiv of 1octyne. Note that this spectrum was collected using solvent suppression. 


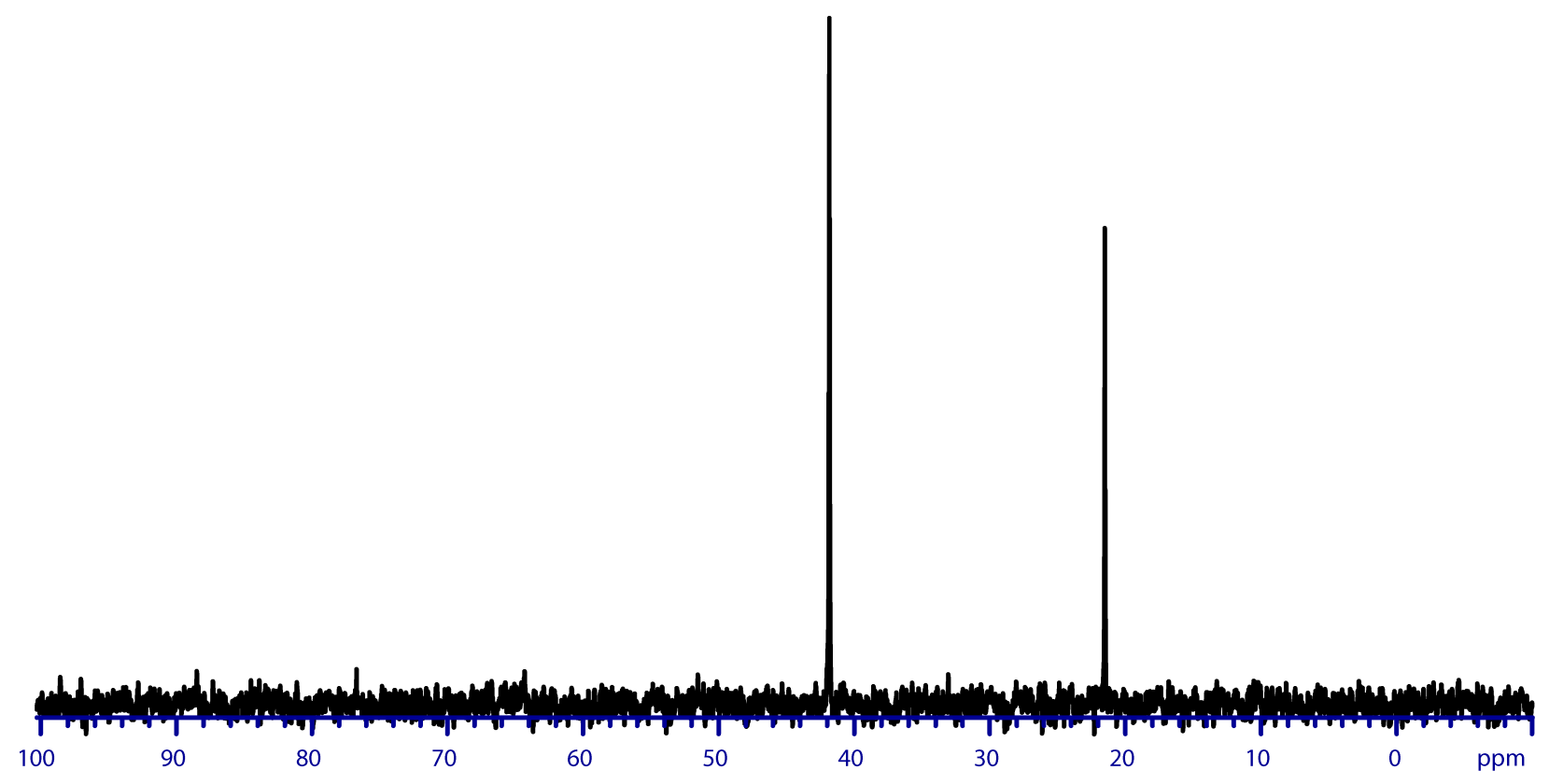

Figure S24. ${ }^{31} \mathrm{P}\left\{{ }^{1} \mathrm{H}\right\} \mathrm{NMR}\left(283 \mathrm{MHz}, \mathrm{C}_{6} \mathrm{H}_{6}\right)$ spectrum of $\mathrm{HRu}\left(\mathrm{CH}_{2} \mathrm{BCatMepi}\right) \mathrm{PPh}_{3}$ and 2 equiv of 1-octyne.

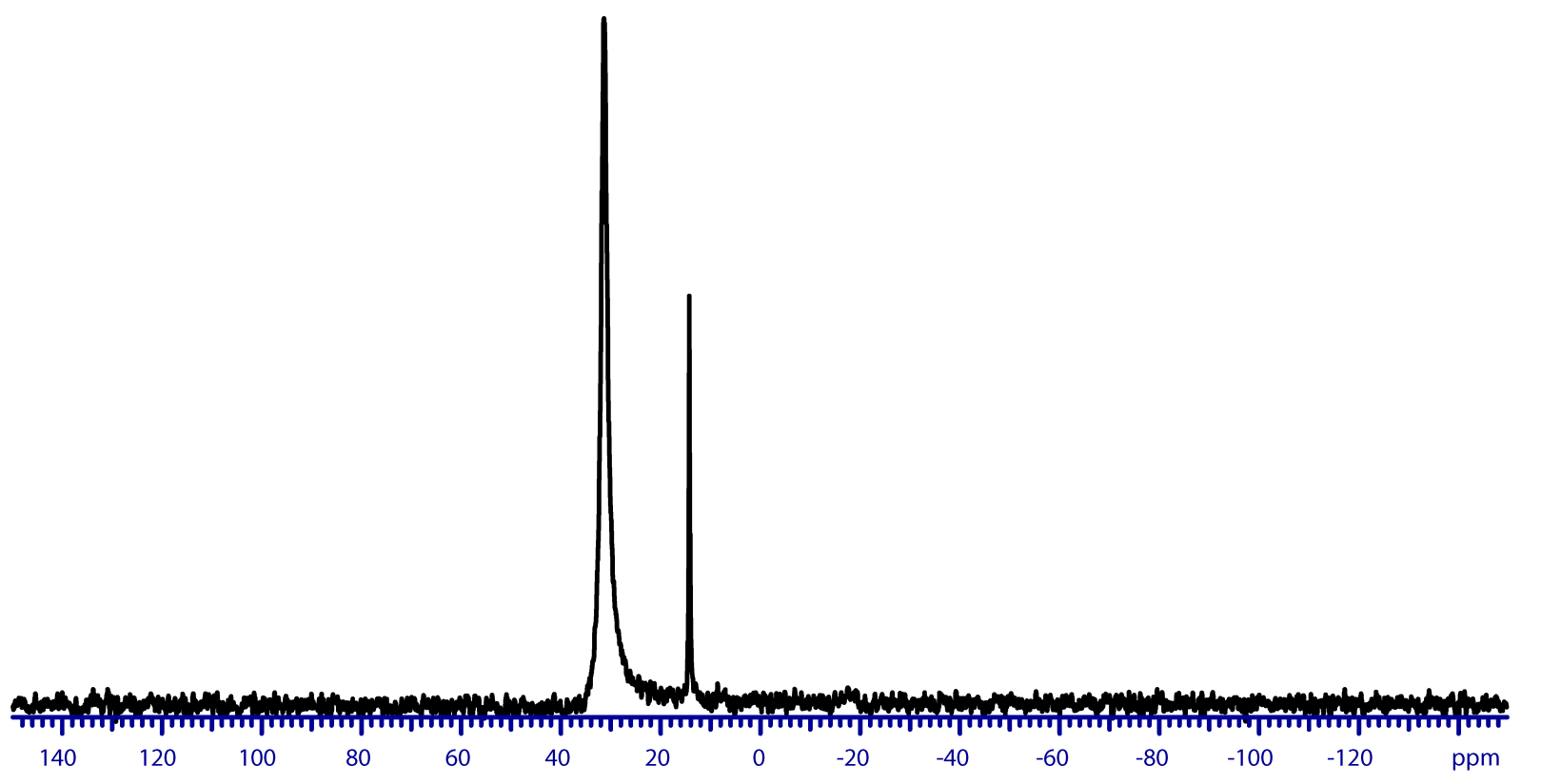

Figure S25. ${ }^{11} \mathrm{~B}\left\{{ }^{1} \mathrm{H}\right\} \mathrm{NMR}\left(160 \mathrm{MHz}, \mathrm{C}_{6} \mathrm{H}_{6}\right)$ spectrum of $\mathrm{HRu}\left(\mathrm{CH}_{2} \mathrm{BCatMepi}\right) \mathrm{PPh}_{3}$ and 2 equiv of 1-octyne. 


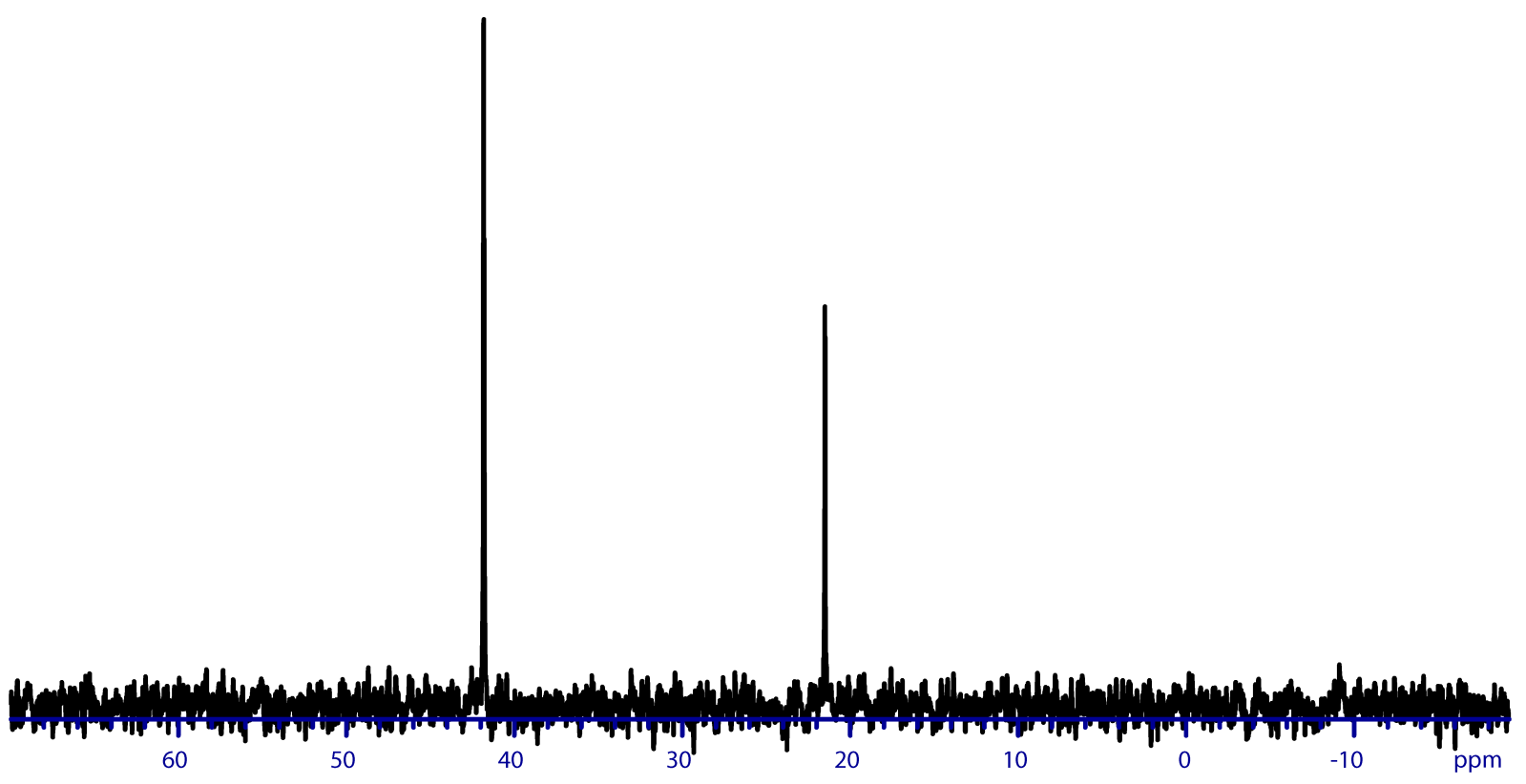

Figure S26. ${ }^{31} \mathrm{P}\left\{{ }^{1} \mathrm{H}\right\} \mathrm{NMR}\left(283 \mathrm{MHz}, \mathrm{C}_{6} \mathrm{H}_{6}\right)$ spectrum of $\mathrm{HRu}\left(\mathrm{CH}_{2} \mathrm{BCatMepi}\right) \mathrm{PPh}_{3}$, 1:1 of 1octene to 1-octyne.

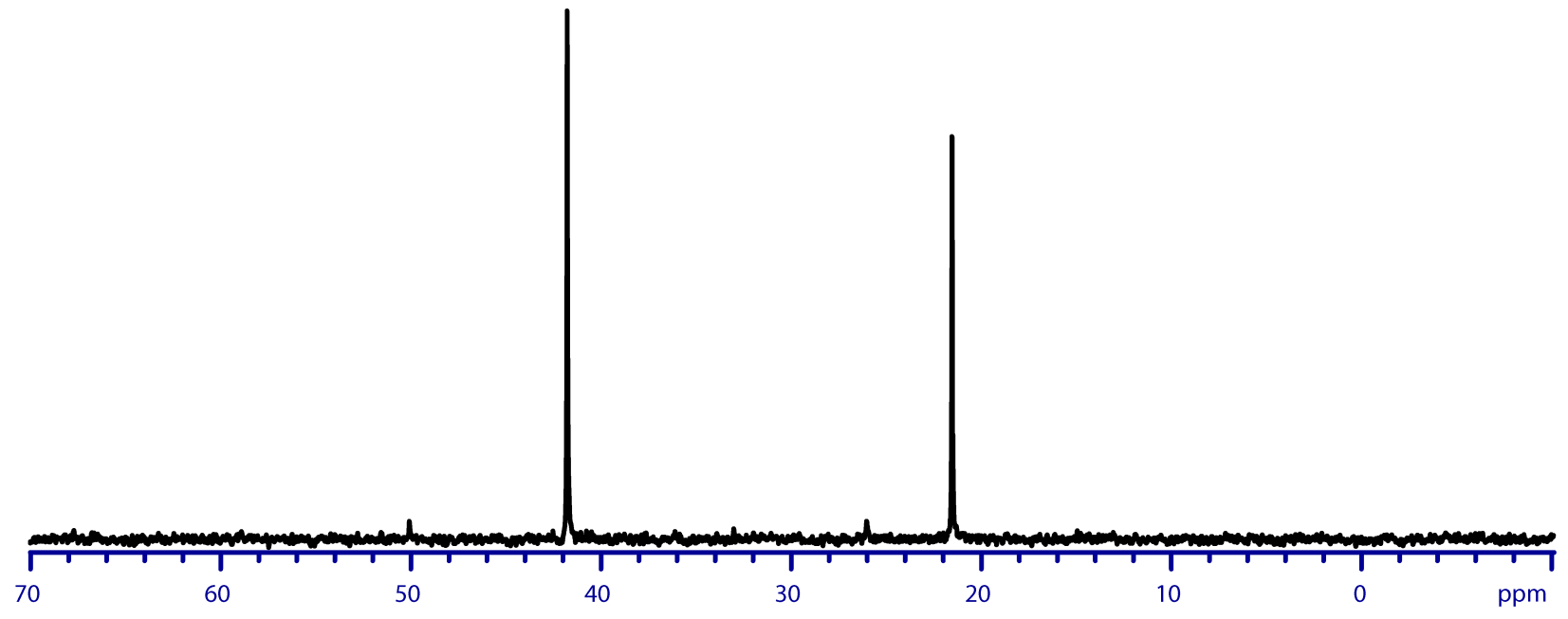

Figure S27. ${ }^{31} \mathrm{P}\left\{{ }^{1} \mathrm{H}\right\}$ NMR $\left(283 \mathrm{MHz}, \mathrm{C}_{6} \mathrm{H}_{6}\right)$ spectrum of $\mathrm{HRu}\left(\mathrm{CH}_{2} \mathrm{BCatMepi}\right) \mathrm{PPh}_{3}$, 100:1 of 1octene to 1-octyne. 


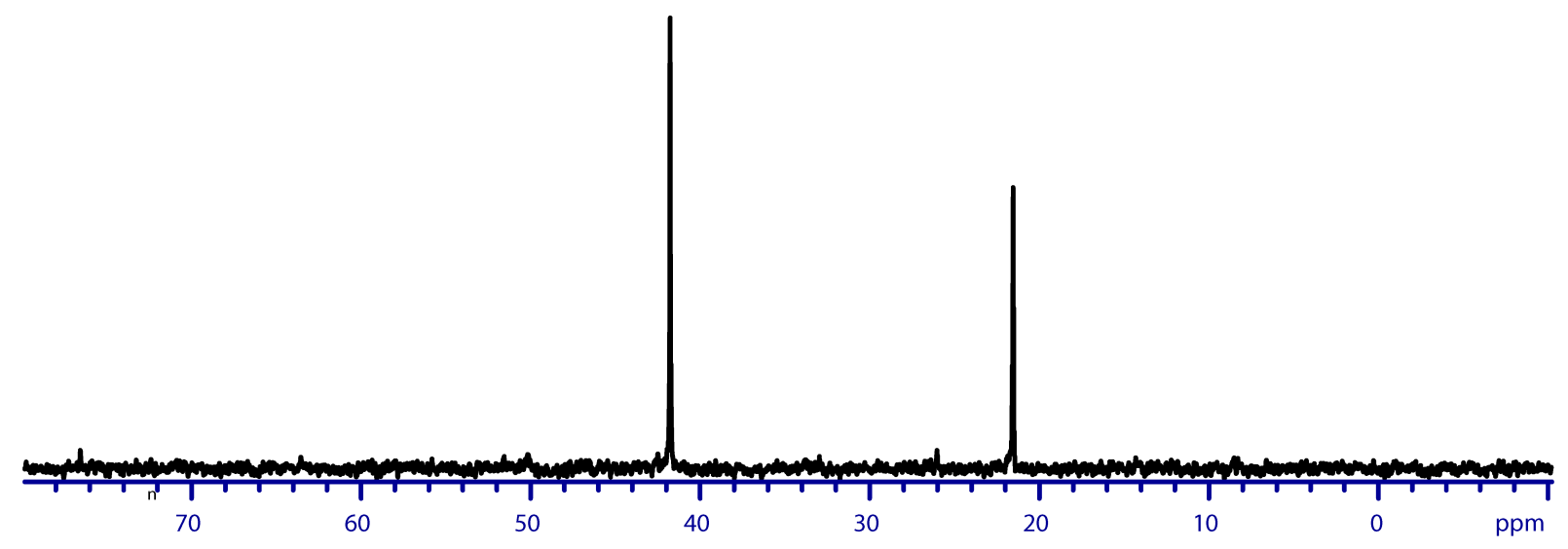

Figure S28. ${ }^{31} \mathrm{P}\left\{{ }^{1} \mathrm{H}\right\} \mathrm{NMR}\left(283 \mathrm{MHz}, \mathrm{C}_{6} \mathrm{H}_{6}\right)$ spectrum of $\mathrm{HRu}\left(\mathrm{CH}_{2} \mathrm{BCatMepi}\right) \mathrm{PPh}_{3}$, 1000:1 of 1octene to 1-octyne. 


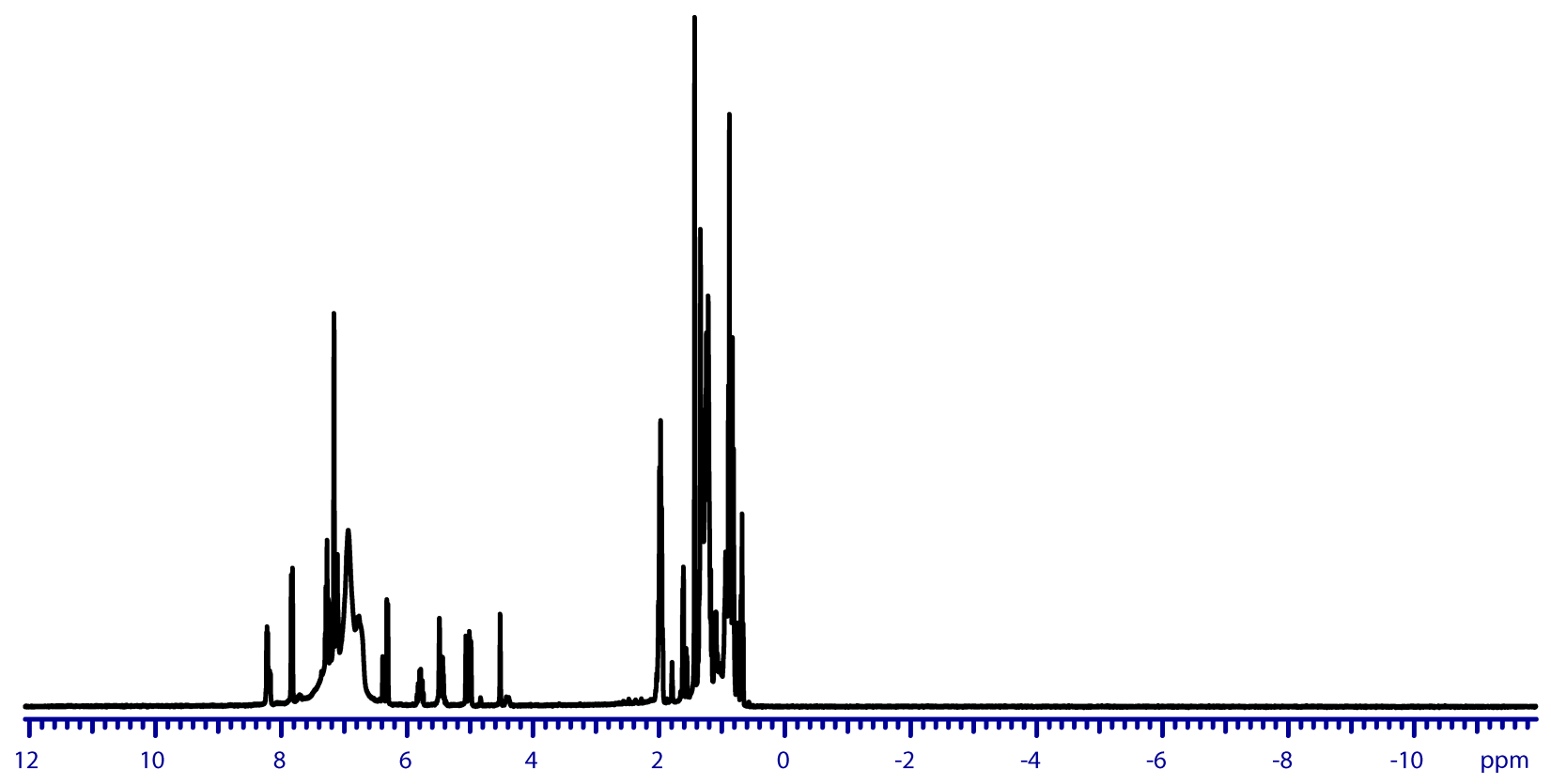

Figure S29. ${ }^{1} \mathrm{H}$ NMR $\left(500 \mathrm{MHz}, \mathrm{C}_{6} \mathrm{D}_{6}\right)$ spectrum of $\mathrm{HRu}(\mathrm{bMepi})\left(\mathrm{PPh}_{3}\right)_{2}$ and 1:1 of 1-octene to 1-octyne.

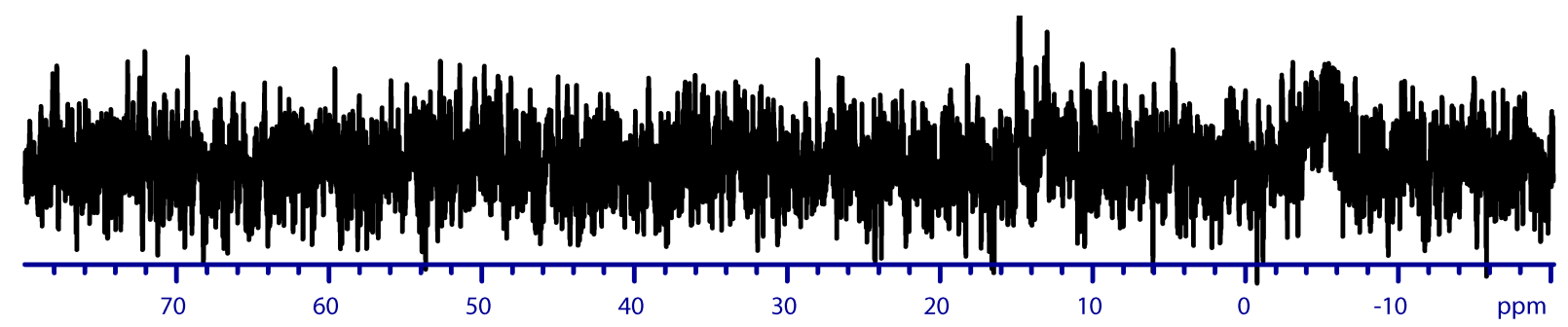

Figure S30. ${ }^{31} \mathrm{P}\left\{{ }^{1} \mathrm{H}\right\}$ NMR (162 MHz, $\left.\mathrm{C}_{6} \mathrm{D}_{6}\right)$ spectrum of $\mathrm{HRu}(\mathrm{bMepi})\left(\mathrm{PPh}_{3}\right)_{2}$ and 1:1 of 1octene to 1-octyne. 


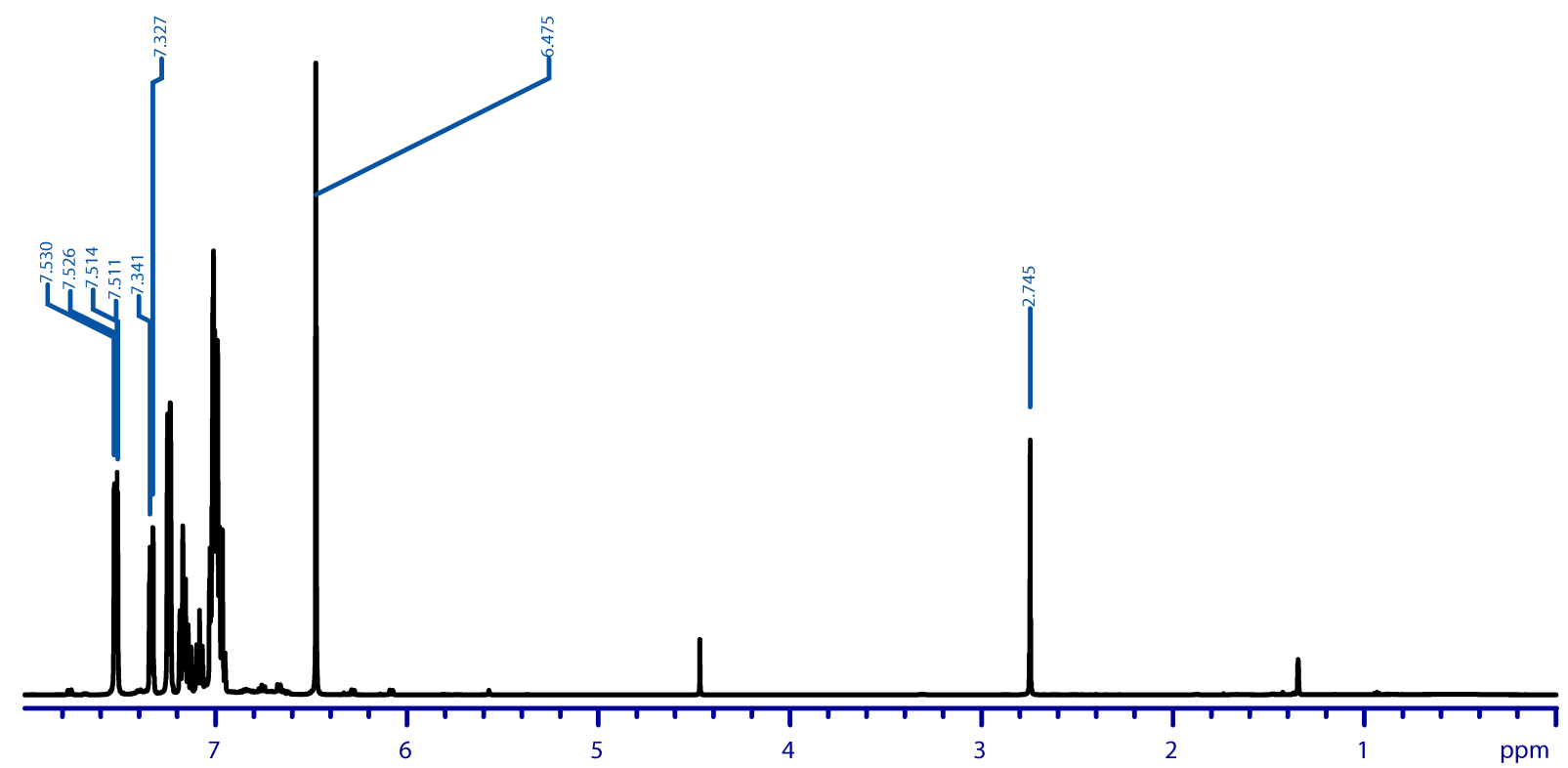

Figure S31. ${ }^{1} \mathrm{H}$ NMR (500 $\left.\mathrm{MHz}, \mathrm{C}_{6} \mathrm{D}_{6}\right)$ spectrum of hydrogenation of diphenylacetylene catalyzed by $\mathrm{HRu}(\mathrm{bMepi})\left(\mathrm{PPh}_{3}\right)_{2}$ after $2 \mathrm{~h}$.

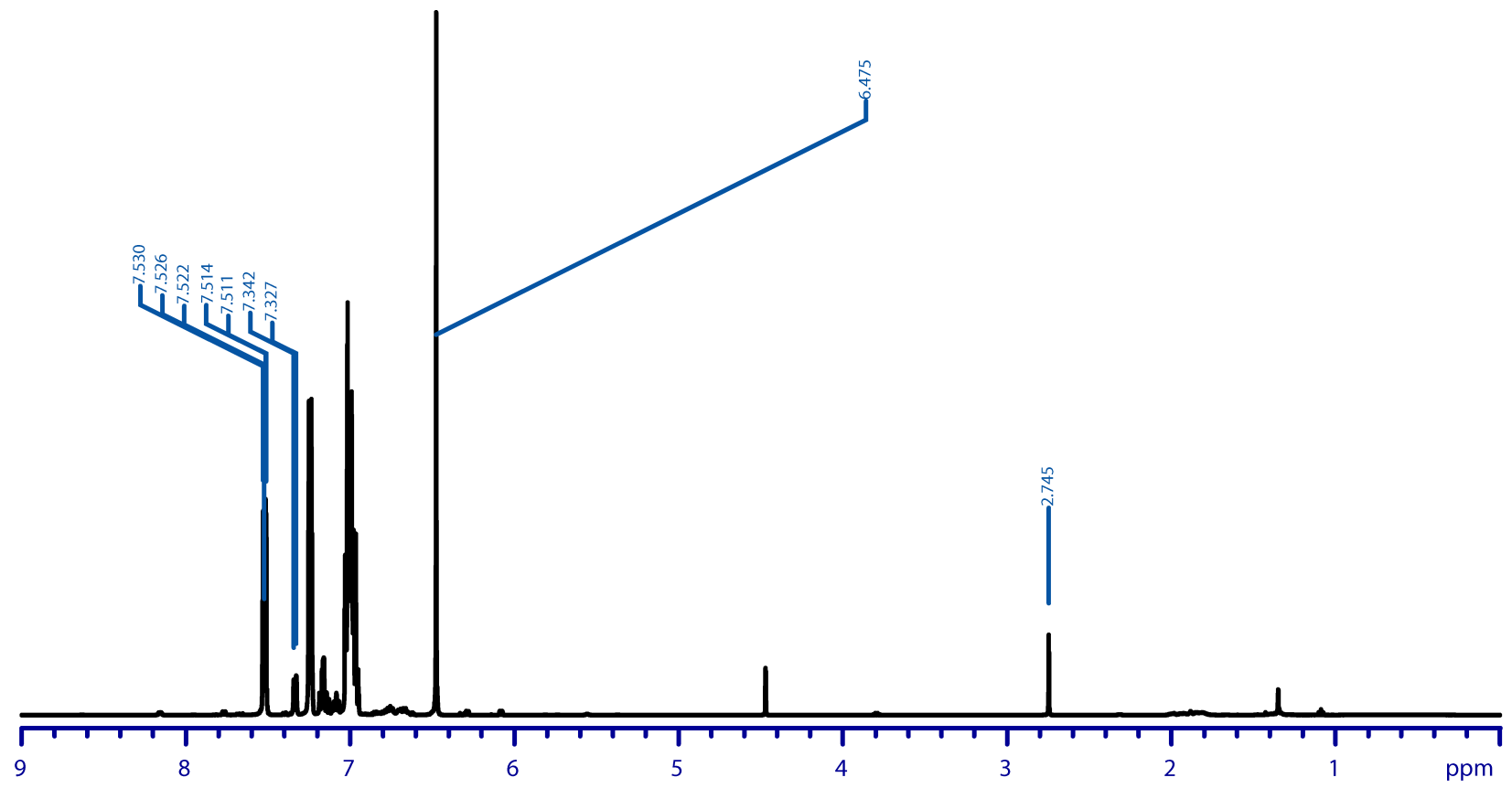

Figure S32. ${ }^{1} \mathrm{H}$ NMR (500 $\left.\mathrm{MHz}, \mathrm{C}_{6} \mathrm{D}_{6}\right)$ spectrum of hydrogenation of diphenylacetylene catalyzed by $\mathrm{HRu}\left(\mathrm{CH}_{2} \mathrm{BCatMepi}\right) \mathrm{PPh}_{3}$ after $2 \mathrm{~h}$. 


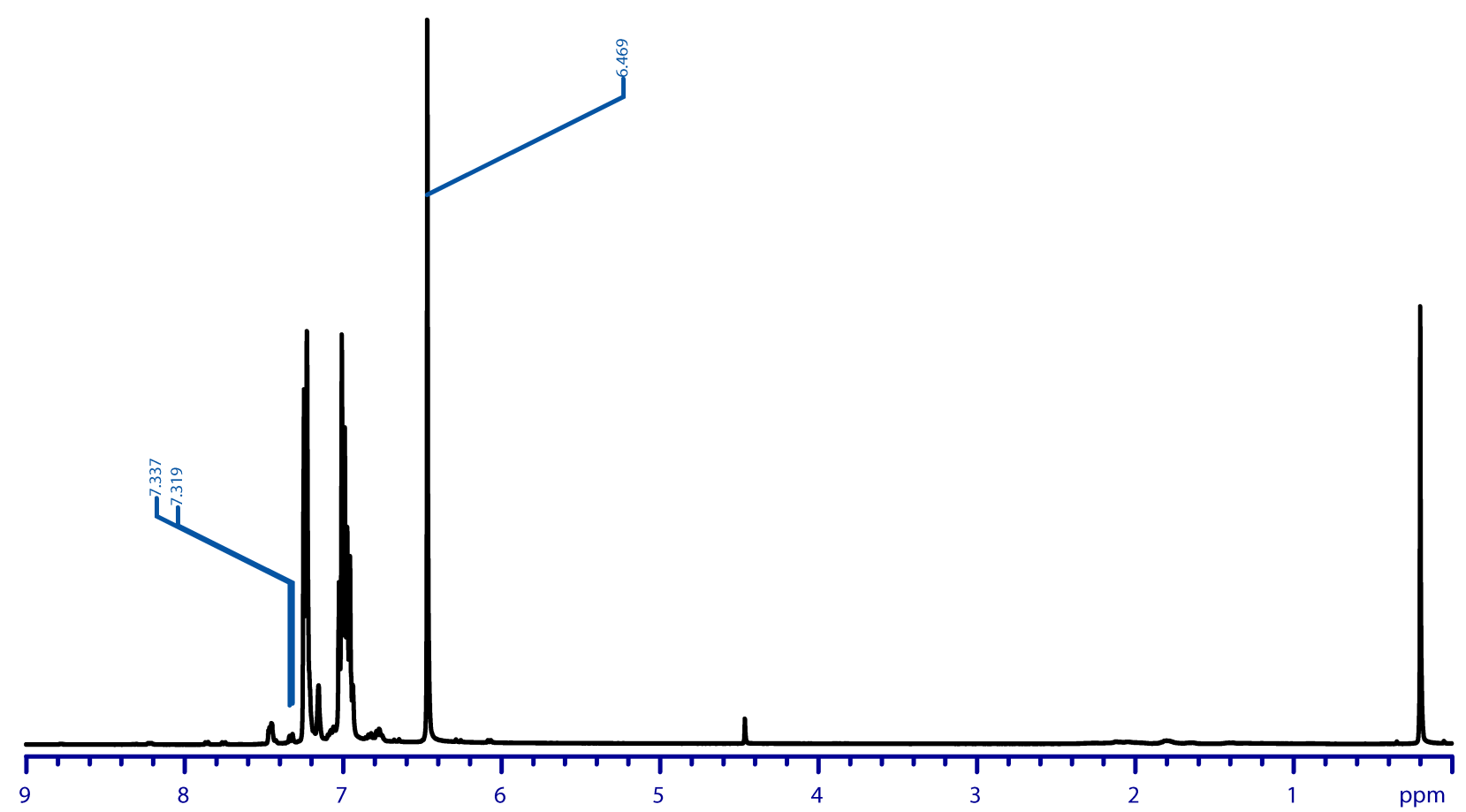

Figure S33. ${ }^{1} \mathrm{H}$ NMR (500 $\left.\mathrm{MHz}, \mathrm{C}_{6} \mathrm{D}_{6}\right)$ spectrum of hydrogenation of diphenylacetylene catalyzed by $\mathrm{Ru}(\mathrm{CH} 9 \mathrm{BBNMepi}) \mathrm{PPh}_{3}$ after $2 \mathrm{~h}$.

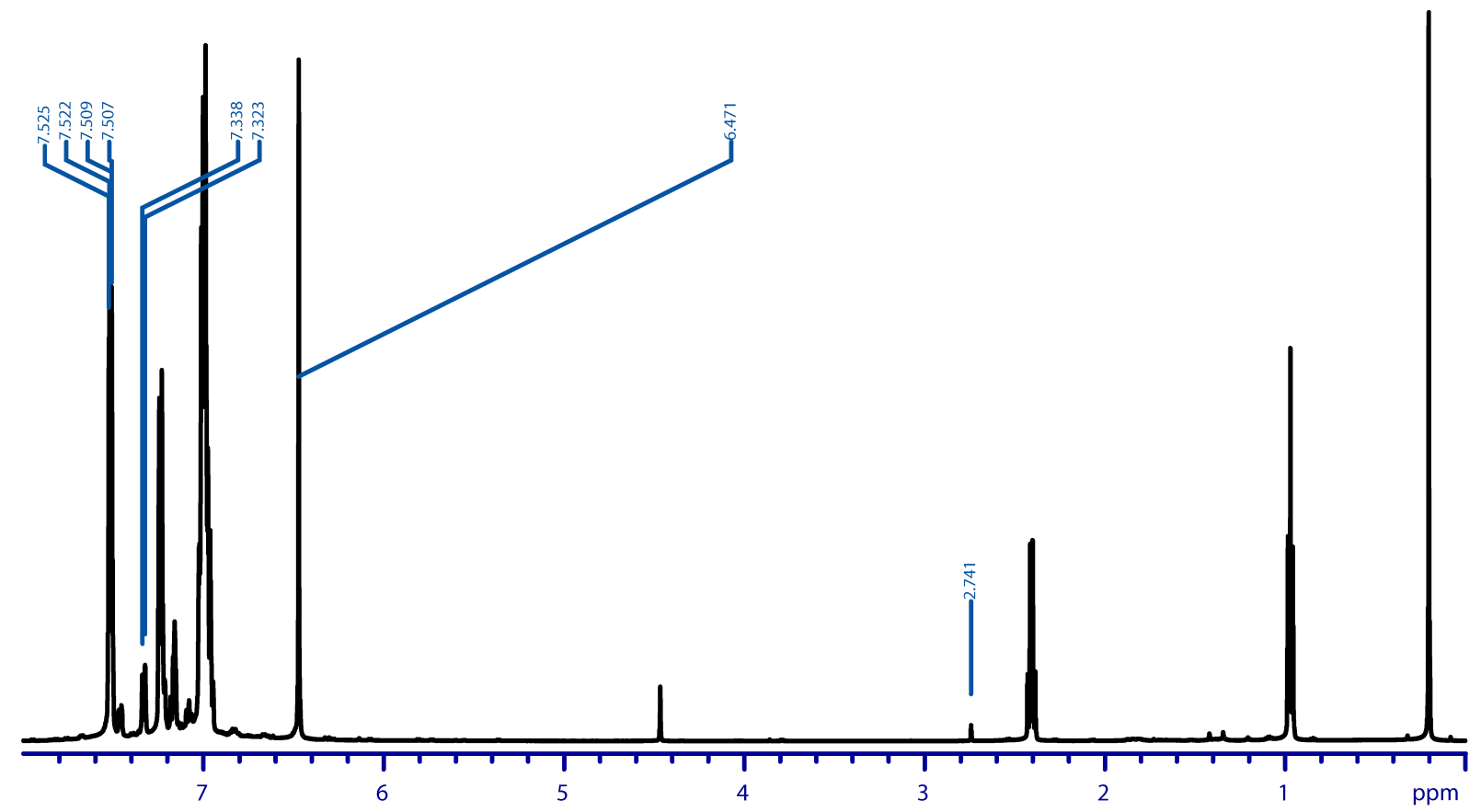

Figure S34. ${ }^{1} \mathrm{H}$ NMR (500 $\left.\mathrm{MHz}, \mathrm{C}_{6} \mathrm{D}_{6}\right)$ spectrum of hydrogenation of diphenylacetylene catalyzed by $\mathrm{Ru}(\mathrm{CH} 9 \mathrm{BBNMepi}) \mathrm{PPh}_{3}$ in the presence of $\mathrm{NEt}_{3}$ after $2 \mathrm{~h}$. 


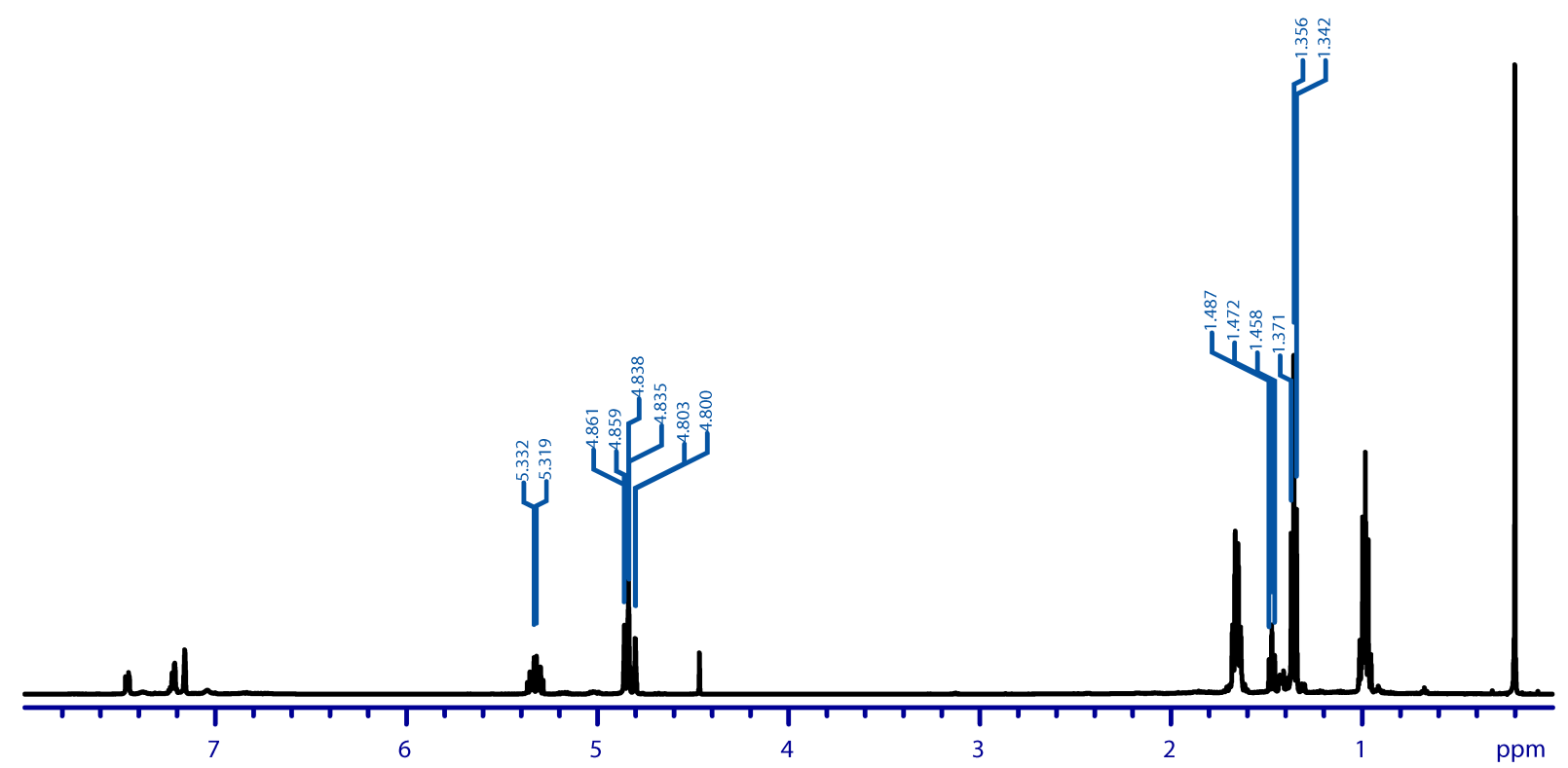

Figure S35. ${ }^{1} \mathrm{H}$ NMR $\left(500 \mathrm{MHz}, \mathrm{C}_{6} \mathrm{D}_{6}\right)$ spectrum of hydrogenation of 5-hexynenitrile catalyzed by $\mathrm{Ru}(\mathrm{CH} 9 \mathrm{BBNMepi}) \mathrm{PPh}_{3}$ after $2 \mathrm{~h}$. For clarity, only the peak values used to identify 5hexynenitrile and 5-hexenenitrile have been marked: $\delta 5.32(\mathrm{~m}, 1 \mathrm{H}, 5$-hexenenitrile), $4.84(\mathrm{t}, 2 \mathrm{H}$, 5-hexenenitrile), 1.47 ( $\mathrm{t}, 2 \mathrm{H}, 5$-hexynenitrile), 1.36 (t, 2H, 5-hexenenitrile). The unlabeled multiplets at $\sim \delta 1$ and $\sim 1.7$ are alkyl signals associated with both the starting material and the product that overlap. 


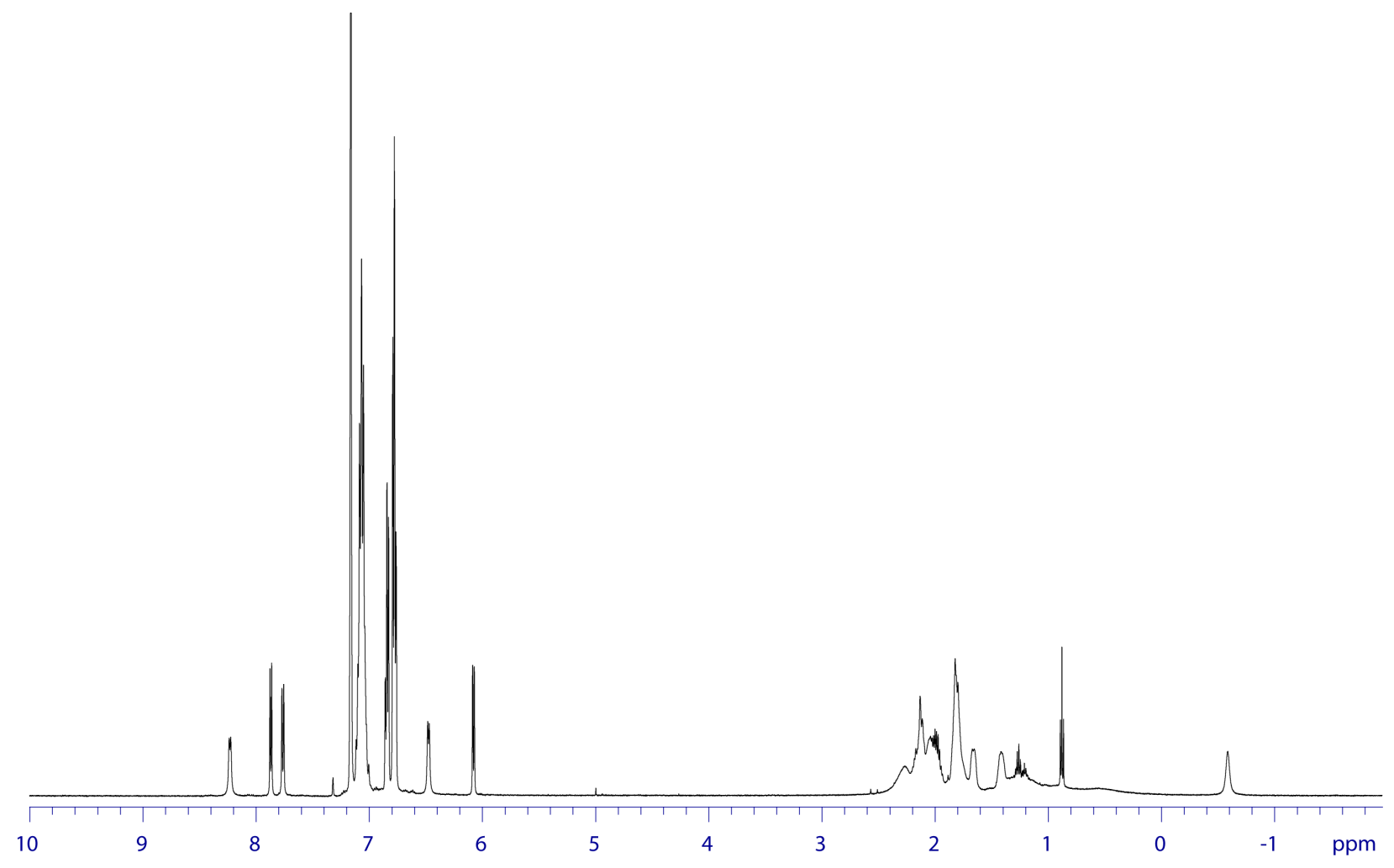

Figure S36. ${ }^{1} \mathrm{H}$ NMR (500 MHz, $\left.\mathrm{C}_{6} \mathrm{D}_{6}\right)$ spectrum of $\mathrm{Ru}(\mathrm{CH} 9 \mathrm{BBNMepi}) \mathrm{PPh}_{3}$.

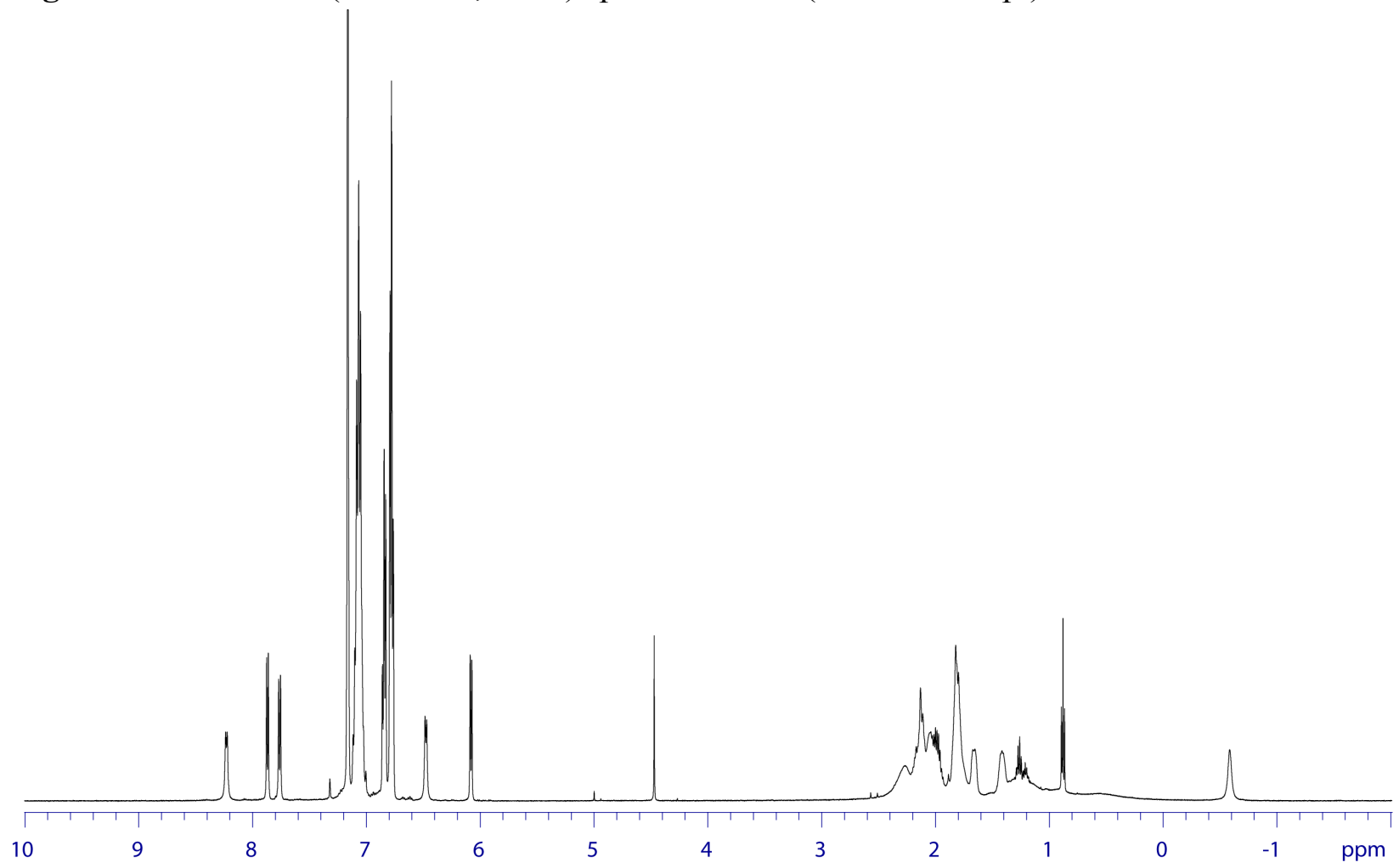

Figure S37. ${ }^{1} \mathrm{H}$ NMR $\left(500 \mathrm{MHz}, \mathrm{C}_{6} \mathrm{D}_{6}\right)$ spectrum of $\mathrm{Ru}(\mathrm{CH} 9 \mathrm{BBNMepi}) \mathrm{PPh}_{3}$ under 30 psig of $\mathrm{H}_{2}$. 


\section{Mechanistic Proposal}

The origin of selective alkyne reduction most likely arises from the acidic character of the pendent boranes, rather than an increased steric profile to direct substrate binding. This preference for alkynes likely emerges from a stronger interaction with the boron atom and is consistent with prior hydroboration studies that similarly demonstrated enhanced rates for alkyne substrates. ${ }^{4}$ Overall, the appended Lewis acid was demonstrated to play a multifunctional role to promote stereoselective alkyne hydrogenation. Following $\mathrm{H}_{2}$ activation, we propose the resulting $\mathrm{Ru}-\mathrm{H}-\mathrm{B}$ intermediate inserts into an alkyne substrate and is assisted by the Lewis acidic borane. $\mathrm{H}_{2}$ addition affords the cis-alkene product and the $\mathrm{Ru}-\mathrm{H}-\mathrm{B}$ species, which re-enters the hydrogenation cycle.

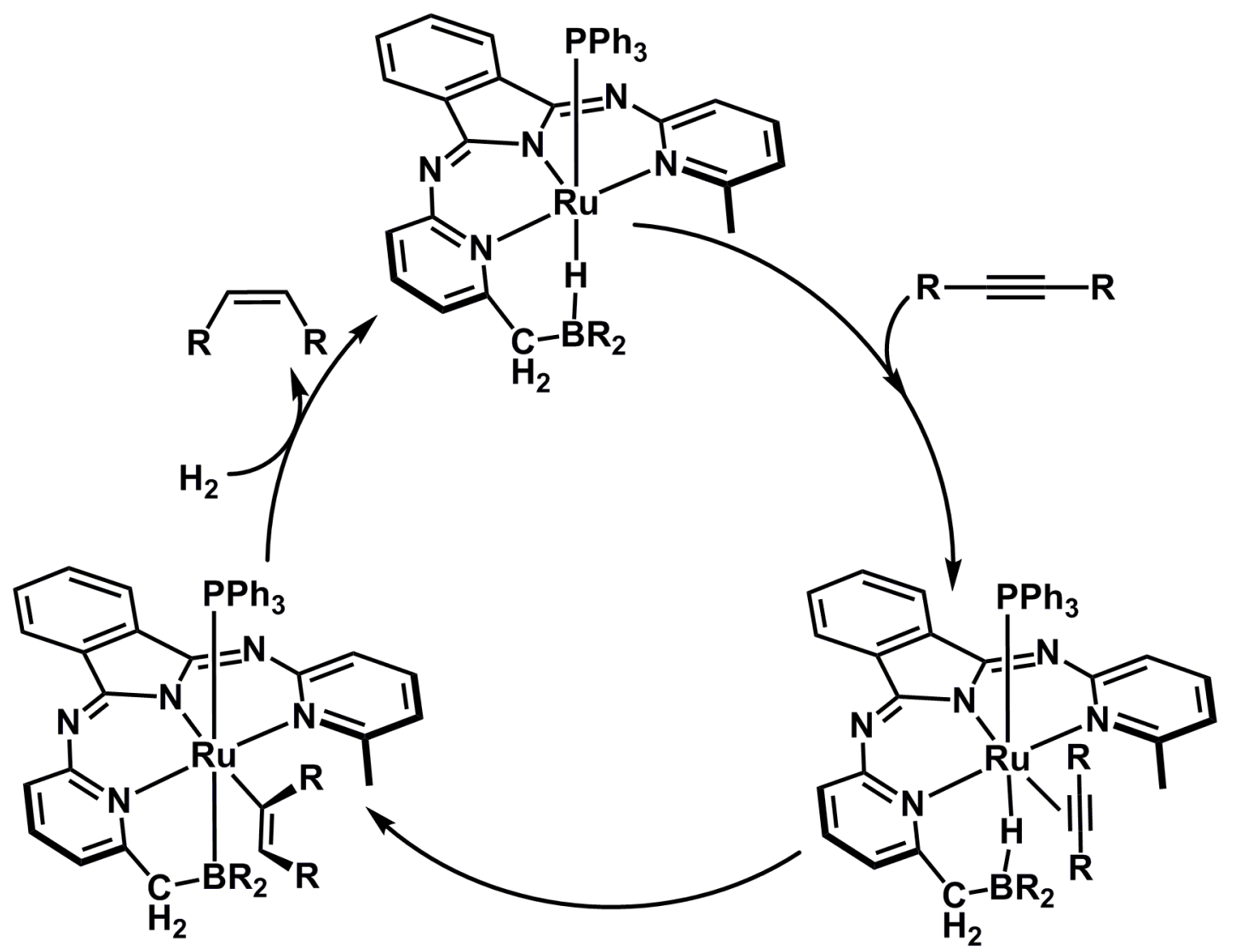

Figure S38. Proposed mechanistic cycle for stereoselective alkyne hydrogenation catalyzed by bifunctional Ru complexes with appended Lewis acids. 


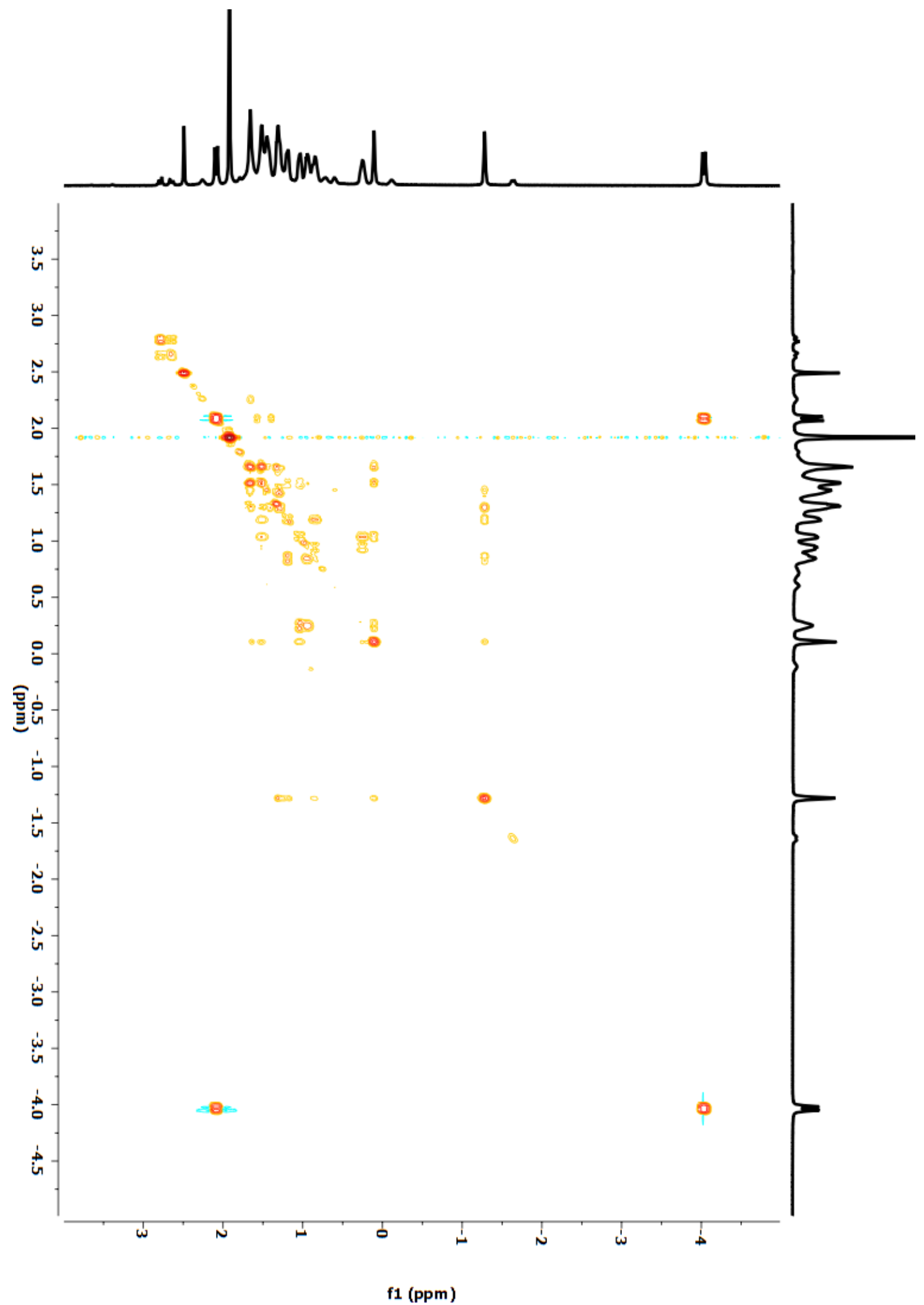

Figure S39. ${ }^{1} \mathrm{H}-\mathrm{gCOSY}\left(500 \mathrm{MHz}, \mathrm{CD}_{2} \mathrm{Cl}_{2},-80{ }^{\circ} \mathrm{C}\right)$ spectrum of $\mathrm{Ru}(\mathrm{CH} 9 \mathrm{BBNMepi}) \mathrm{PPh}_{3}$. 


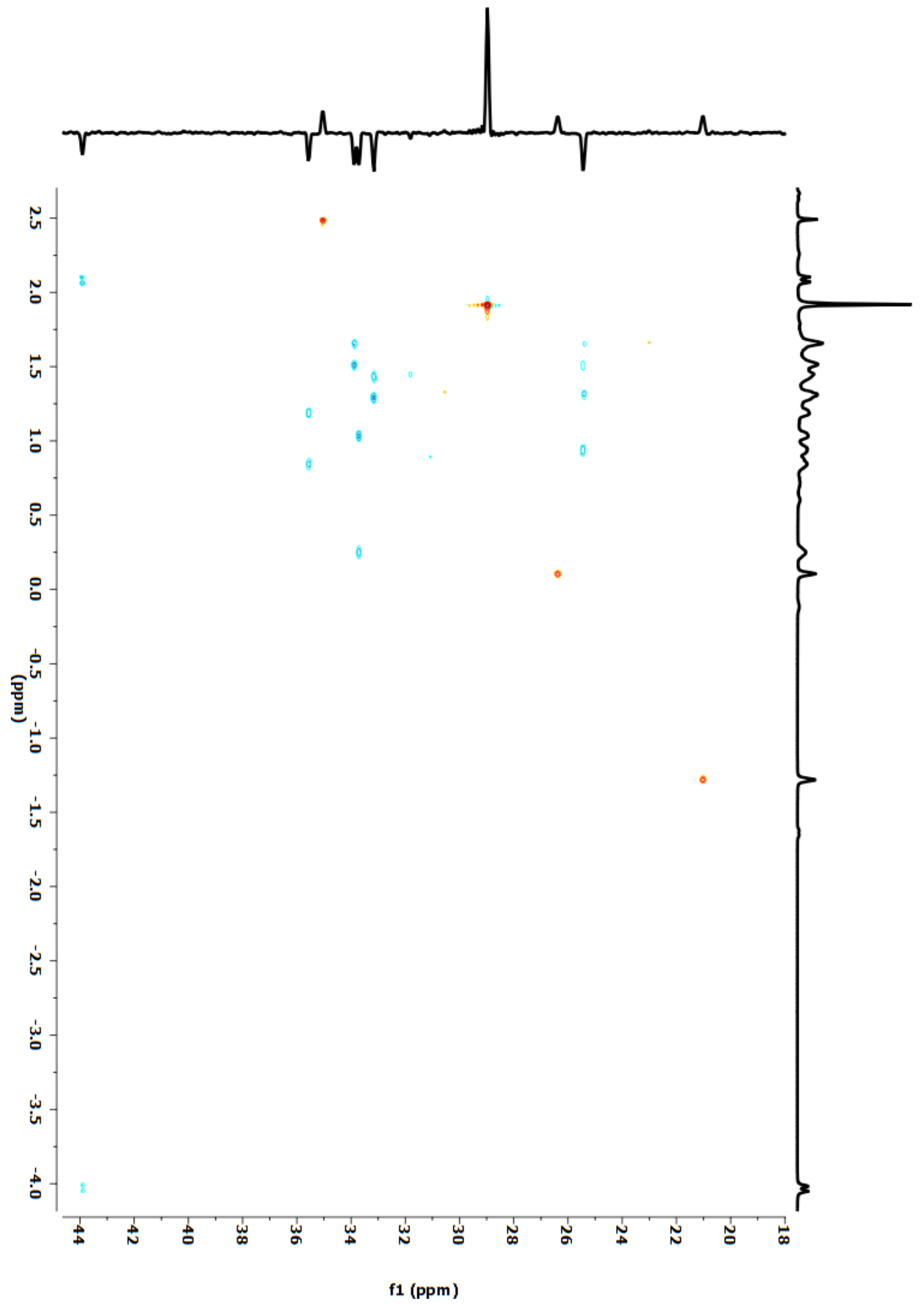

Figure S40. $\quad{ }^{1} \mathrm{H}-{ }^{13} \mathrm{CgHSQCAD} \quad\left(500 \quad \mathrm{MHz}, \quad \mathrm{CD}_{2} \mathrm{Cl}_{2}, \quad-80 \quad{ }^{\circ} \mathrm{C}\right) \quad$ spectrum of $\mathrm{Ru}(\mathrm{CH} 9 \mathrm{BBNMepi}) \mathrm{PPh}_{3}$. 


\section{9-BBN fragment analyzed by $2 D N M R$}

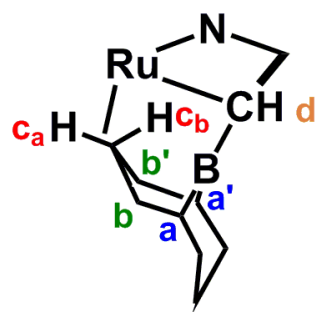

A ${ }^{1} \mathrm{H}-{ }^{13} \mathrm{C}$ gHSQCAD experiment was used to analyze the connectivity and coordination mode of the $\mathrm{CH}-9-\mathrm{BBN}$ moiety. The crosspeaks in the 2D spectrum (Figure S40), confirm that the two Hs resonating at $2.14\left(\mathrm{c}_{\mathrm{b}}\right)$ and $-3.9 \mathrm{ppm}\left(\mathrm{c}_{\mathrm{a}}\right)$ are on the same carbon. Furthermore, the phase of the crosspeaks was used to identify the $\mathrm{H}$ signals in the ${ }^{1} \mathrm{H}$ NMR spectrum that are of the type $\mathrm{CHR}_{3}$ $\left(a, a^{\prime}, d\right)$. A COSY experiment (Figure S39) revealed that $c_{a}$ and $c_{b}$ do not couple to any $\mathrm{CHR}_{3}$ protons $\left(a, a^{\prime}, d\right)$ but do couple with another set of $\mathrm{CH}_{2} \mathrm{R}_{2}$ protons. These data suggest that $\mathrm{c}_{\mathrm{a}}$ and $\mathrm{c}_{\mathrm{b}}$ belong to a wingtip carbon on the 9-BBN ring.

We note that the chair-type confirmation is common, and a search of the CSD revealed more than 35 examples of this chair-type conformation (for select examples see Chem. Commun., 2007, 4662-4664 and Eur. J. Inorg. Chem., 2011, 5414-5421). 


\section{References}

1. Tseng, K.-N. T.; Kampf, J. W.; Szymczak, N. K. Organometallics 2013, 32, 2046.

2. Tseng, K.-N. T.; Kampf, J. W.; Szymczak, N. K. ACS Catal. 2015, 5, 5468.

3. Ohmiya, H.; Shido, Y.; Yoshida, M.; Sawamura, M. Chem. Lett. 2011, 40, 928.

4. Chernichenko, K.; Madarasz, A.; Papai, I.; Nieger, M.; Leskelae, M.; Repo, T. Nat. Chem. 2013, 5, 718.

5. Hale, L. V. A.; Malakar, T.; Tseng, K. T.; Zimmerman, P. M.; Paul, A.; Szymczak, N. K. ACS Catal. 2016, 6, 4799-4813.

6. Cassen, A.; Gloaguen, Y.; Vendier, L.; Duhayon, C.; Poblador-Bahamonde, A.; Raynaud, C.; Clot, E.; Alcaraz, G.; Sabo-Etienne, S. Angew. Chem., Int. Ed. 2014, 53, 7569. 